\title{
Application of array comparative genomic hybridization in 256 patients with developmental delay or intellectual disability
}

\author{
Magdalena Bartnik • Beata Nowakowska • Katarzyna Derwińska • \\ Barbara Wiśniowiecka-Kowalnik • Marta Kędzior • Joanna Bernaciak • \\ Kamila Ziemkiewicz • Tomasz Gambin • Maciej Sykulski • Natalia Bezniakow • \\ Lech Korniszewski • Anna Kutkowska-Kaźmierczak • Jakub Klapecki • \\ Krzysztof Szczałuba • Chad A. Shaw • Tadeusz Mazurczak • Anna Gambin • \\ Ewa Obersztyn • Ewa Bocian • Pawel Stankiewicz
}

Received: 8 August 2013 / Revised: 17 October 2013 / Accepted: 20 October 2013 /Published online: 3 December 2013

(C) The Author(s) 2013. This article is published with open access at Springerlink.com

\begin{abstract}
We used whole-genome exon-targeted oligonucleotide array comparative genomic hybridization (array CGH) in a cohort of 256 patients with developmental delay (DD)/
\end{abstract}

Electronic supplementary material The online version of this article (doi:10.1007/s13353-013-0181-x) contains supplementary material, which is available to authorized users.

M. Bartnik $\cdot$ B. Nowakowska $\cdot$ K. Derwińska $\cdot$

B. Wiśniowiecka-Kowalnik • M. Kędzior • J. Bernaciak •

K. Ziemkiewicz • N. Bezniakow • A. Kutkowska-Kaźmierczak •

J. Klapecki $\cdot$ K. Szczałuba $\cdot$ T. Mazurczak $\cdot$ E. Obersztyn •

E. Bocian $\cdot$ P. Stankiewicz

Department of Medical Genetics, Institute of Mother and Child,

Warsaw, Poland

T. Gambin

Institute of Computer Science, Warsaw University of Technology,

Warsaw, Poland

\section{Sykulski}

Institute of Informatics, University of Warsaw, Warsaw, Poland

\section{Korniszewski}

Genetic Counseling Unit, Institute of Physiology and Pathology of Hearing, World Hearing Center, Warsaw, Poland

C. A. Shaw $\cdot$ P. Stankiewicz $(\bowtie)$

Department of Molecular and Human Genetics, Baylor College of Medicine, One Baylor Plaza, Rm. R809, Houston, TX 77030, USA

e-mail: pawels@bcm.edu

P. Stankiewicz

e-mail: pawel.stankiewicz@imid.med.pl

A. Gambin

Mossakowski Medical Research Centre, Polish Academy of

Sciences, Warsaw, Poland intellectual disability (ID) with or without dysmorphic features, additional neurodevelopmental abnormalities, and/or congenital malformations. In 69 patients, we identified 84 nonpolymorphic copy-number variants, among which 41 are known to be clinically relevant, including two recently described deletions, 4q21.21q21.22 and 17q24.2. Chromosomal microarray analysis revealed also 15 potentially pathogenic changes, including three rare deletions, $5 \mathrm{q} 35.3,10 \mathrm{q} 21.3$, and 13q12.11. Additionally, we found 28 copy-number variants of unknown clinical significance. Our results further support the notion that copy-number variants significantly contribute to the genetic etiology of DD/ID and emphasize the efficacy of the detection of novel candidate genes for neurodevelopmental disorders by whole-genome array CGH.

Keywords Copy-number variation · Microdeletion . Microduplication · Chromosomal microarray analysis

\section{Introduction}

Developmental delay (DD)/intellectual disability (ID), characterized by a significant impairment of cognitive and adaptive functions, affects $1-3 \%$ of the general population (Harris 2006; Maulik et al. 2011), with the majority of affected individuals remaining without proper diagnosis (Rauch et al. 2006; Pfundt and Veltman 2012). The etiology of DD/ID is heterogeneous, with both genetic and environmental contribution (Grayton et al. 2012). In addition to Mendelian DD/ID, one of the most common causes are microscopically visible chromosomal aberrations and submicroscopic copy- 
number variants (CNVs) (Morrow 2010; Regier et al. 2010). A high-resolution G-banded karyotype reveals chromosome abnormalities in $3-5 \%$ of patients with idiopathic DD/ID (Shevell et al. 2003), and molecular cytogenetic analyses, e.g., fluorescent in situ hybridization (FISH) in the subtelomeric regions, provides diagnosis in an additional 3$6 \%$ of cases (Koolen et al. 2004; Ravnan et al. 2006). In the past few years, application of chromosomal microarray analysis (CMA), including array comparative genomic hybridization (array CGH) and single-nucleotide polymorphism (SNP) arrays, has revolutionized the clinical diagnostics in patients with idiopathic DD/ID (Cooper et al. 2011; Kaminsky et al. 2011; Girirajan et al. 2012). Recently, it has been proven that CMA should be a first-tier clinical diagnostic test for individuals with DD, ID, autism spectrum disorders, and dysmorphic features (Miller et al. 2010; Battaglia et al. 2013). Clinically relevant CNVs, ranging in size from megabases to kilobases (Rodriguez-Revenga et al. 2007), have been detected in $~ 10$ $20 \%$ of cases (Menten et al. 2006; Stankiewicz and Beaudet 2007; Koolen et al. 2009) and have led to the identification of several novel microdeletions and microduplications associated with DD/ID (Slavotinek 2008; Vissers and Stankiewicz 2012), e.g., involving chromosomes 1q41q42, 9q22.3, 15q13.3, 15q24, 16p11.2, and 17q21.31 (Koolen et al. 2006; Redon et al. 2006; Sharp et al. 2006; Shaw-Smith et al. 2006; Ballif et al. 2007; Shaffer et al. 2007; Sharp et al. 2007, 2008). Refinement of the critical region of a known syndrome by the identification of atypical deletion (Cooper et al. 2011) may facilitate the detection of a dosage-sensitive gene(s) related to ID (Vissers et al. 2010); however, there are many CNVs for which the clinical significance may still remain unknown (Rodriguez-Revenga et al. 2007). Recently, clinical and biological interpretation of those variants and their genotypephenotype correlation enabled the generation of a human genome morbid map (Cooper et al. 2011).

Here, we present the results of the application of genome-wide array CGH in a cohort of 256 patients with $\mathrm{DD} / \mathrm{ID}$, dysmorphic features, congenital malformations, or additional neurodevelopmental abnormalities. We identified 84 non-polymorphic CNVs in 69 patients, including 41 clinically relevant CNVs, 15 potentially novel pathogenic genetic loci for DD/ID, and, additionally, $28 \mathrm{CNVs}$ of unknown clinical significance, likely to be non-pathogenic changes.

\section{Materials and methods}

Patients

We studied 256 patients with DD/ID, dysmorphic features, congenital malformations, or additional neurodevelopmental abnormalities. Among them, 234 patients had normal GTG banding analysis with at least 550-band resolution and 46 patients had a negative fragile $\mathrm{X}$ testing.

We provide a detailed clinical description of five patients (Supplementary material) and discuss their genotype-phenotype correlations. Patients 23 and 34 have de novo CNVs that we believe are pathogenic, and patients 45, 50, and 51 have potentially pathogenic CNVs.

\section{DNA isolation}

Genomic DNA was extracted from peripheral blood cells using a Puregene DNA Blood Kit (Qiagen, Gentra Systems, Minneapolis, MN), according to the manufacturer's protocol. The reference DNA samples were obtained from phenotypically normal male and female controls.

\section{Array CGH}

Custom-designed exon-targeted clinical array CGH was performed using $105 \mathrm{~K} \mathrm{V7.4} \mathrm{and} \mathrm{180K} \mathrm{V8.0} \mathrm{or} \mathrm{V8.1} \mathrm{microarrays}$ designed in the Medical Genetics Laboratories at Baylor College of Medicine (BCM) (http://www.bcm.edu/geneticlabs/ cma/tables.html) in cooperation with the Department of Medical Genetics at the Institute of Mother and Child and manufactured by Agilent Technologies (Santa Clara, CA). V7.4 consisted of 105,000 oligonucleotides having genome-wide coverage with an average resolution of $30 \mathrm{~kb}$, while V8.0 and V8.1 OLIGO $(180 \mathrm{~K})$ arrays have genome-wide coverage as well as exon coverage for over 1,700 known or candidate disease genes with an average of 4.2 oligos per exon and intronic gaps no larger than $10 \mathrm{~kb}$ (Boone et al. 2010). The microarray used in this study does not contain SNP probes and it does not detect regions of absence of heterozygosity (AOH). The results of studies using the updated version of this array were recently published by the BCM (Wiszniewska et al. 2013). Digestion, labeling, and hybridization were performed following the manufacturer's instructions. The BCM web-based software platform and the homebrew IMiD-web2py software were used for chromosomal microarray analysis. All genomic coordinates are based on the March 2006 assembly of the reference genome (NCBI36/hg18). To verify the rearrangements identified by array CGH, depending on CNVs size, we used GTG banding and FISH. When available, blood samples were obtained from patients' parents, and CNV inheritance was investigated.

\section{Karyotype analysis}

GTG banding analysis was performed according to the standard protocol in peripheral blood lymphocytes. The metaphases with 550-band resolution were analyzed in cases with $\mathrm{CNVs} 5 \mathrm{Mb}$ or greater in size. 
Fluorescent in situ hybridization (FISH) analysis

Confirmatory FISH experiments were performed to verify the presence of CNVs ranging in size from $150 \mathrm{~kb}$ to $5 \mathrm{Mb}$. FISH analyses were performed by standard procedures in phytohemagglutinin-stimulated peripheral blood lymphocytes using probes derived from bacterial artificial chromosomes. When available, blood samples were obtained from the patient's parents and the origin of the identified CNVs was studied using FISH with the same probes.

\section{Results}

A total of 84 non-polymorphic CNVs were found in 69 of 256 patients studied. We divided the detected CNVs into three groups. The first group contains $41 \mathrm{CNVs}$ considered to be clinically relevant (i.e., pathogenic for DD/ID) (Table 1). This group includes 18 imbalances greater than $5 \mathrm{Mb}$ in size identified in 17 patients (not seen in standard cytogenetic studies) and 23 submicroscopic CNVs. In three cases, two pathogenic CNVs were detected: patient 9 had an unbalanced translocation $\operatorname{der}(10) \mathrm{t}(10 ; 20)(\mathrm{q} 26.2 ; \mathrm{q} 13.33)$; patient 10 had a terminal duplication $\operatorname{dup}(11)(\mathrm{p} 15)$ and a terminal deletion del(11)(q24), most likely a recombination product of a pericentromeric inversion inv(11)(p15q24); and patient 12 had a large $15 \mathrm{q} 13.3 \mathrm{q} 14$ deletion in addition to the wellknown recurrent microdeletion 16p13.11. We also identified two mosaic trisomies of chromosome 9 (pt 15 and pt 16) and monosomy of chromosome 7 in a 1-year-old patient (pt 17) with DD and combined immunodeficiency. Among 23 submicroscopic CNVs, we identified known recurrent rearrangements, e.g., deletions 15q11.2, 16p13.3, 17q11.2, and $17 \mathrm{q} 21.31$ and different-sized non-recurrent CNVs, e.g., deletions $1 \mathrm{p} 36.31 \mathrm{p} 36.33,1 \mathrm{q} 43 \mathrm{q} 44,5 \mathrm{q} 14.3,6 \mathrm{q} 25$, and 10q24.32, as well as duplications 3p21.1 and 19p13.3. In this group, we also identified two recently described microdeletions at chromosomes $4 \mathrm{q} 21$ and 17q24.2.

The second group consists of $15 \mathrm{CNV}$ s potentially pathogenic for DD/ID (Table 2). In three cases, we identified rare CNVs: two de novo deletions at 13q12.11 and 5q35.3, and in one patient, a deletion at $10 \mathrm{q} 21.3$ and a duplication at 19p13.42p13.43.

In the third group, we classified 28 deletions and duplications of unknown clinical significance, some of which are likely non-pathogenic for DD/ID (Tables 1, 2, and 3). We found four individuals with BP1/BP2 duplication at $15 \mathrm{q} 11.2$ that is frequently found in the general population and considered as a benign event.

Moreover, we present the cumulative data of the studied cohort (Table 4), including patients with normal array CGH results (Supplementary Table 1).

\section{Discussion}

To determine the phenotypic consequences of the 84 identified non-polymorphic CNVs, we considered their type (deletion vs. duplication), size, gene content, inheritance pattern, and the available clinical or genomic database information. We classified them into three groups: $41(16.0 \%)$ known well-recognized causative for DD/ID, 15 (5.85\%) novel potentially pathogenic, and $28(10.9 \%)$ variants of unknown clinical significance (Tables 1, 2, and 3). The first group includes CNVs responsible for the well-known diseases and syndromes or published genomic imbalances that were considered as clinically relevant for DD/ID. The second group consists of novel CNVs potentially causative for DD/ID and includes changes that were not previously reported to be associated with DD/ID but contain genes that may contribute to our patients' phenotypes. Moreover, we compared CNVs classified as the second group with the publically available databases. The same or similar sized aberrations were not found in the Database of Genomic Variants (DGV, http:// projects.tcag.ca/variation/), Database of Chromosomal Imbalance and Phenotype in Humans Using Ensembl Resources (DECIPHER, http://decipher.sanger.ac.uk), or the International Standards for Cytogenomic Arrays database (ISCA, https://www.iscaconsortium.org). In the third group of variants of unknown clinical significance, we classified $\mathrm{CNVs}$ that contain genes that were not previously associated with DD/ID, are frequently found in the general population, or were inherited from a healthy parent.

From the second group of potentially pathogenic CNVs, we selected for the detailed description three novel rare, probably pathogenic, deletions containing candidate genes that may contribute to the abnormal phenotypes observed in our patients. Furthermore, in two of those cases (pt 51 and pt 45), deletions arose de novo that support their potential pathogenicity.

A de novo $\sim 250-\mathrm{kb}$ deletion in 13q12.11, encompassing only two genes, PSPC1 (paraspeckle component 1; MIM 612408) and ZMYM5 (zinc finger, MYM-type 5), was identified in pt 51 with remarkably delayed psychomotor development, muscle hypotonia, unilateral microphtalmia with ptosis, congenital eye malformation, and facial dysmorphic features (Fig. 1a, d). Interstitial deletions of $13 \mathrm{q} 12.11$ are very rare (Der Kaloustian et al. 2011; Tanteles et al. 2011). Tanteles et al. (2011) described a de novo 2.9-Mb 13q12.11 deletion of 19 genes, including PSPC1 and ZMYM5, in a patient with near normal development and intellect but with scaphocephaly and facial dysmorphism. PSPC1 encodes a nucleolar protein that localizes to punctate subnuclear structures close to splicing speckles, known as paraspeckles. Paraspeckles may function in the control of gene expression via an RNA nuclear retention mechanism. Functionally, PSPC1 was proposed to participate in the regulation of transcription (Passon et al. 2011). ZMYM5 


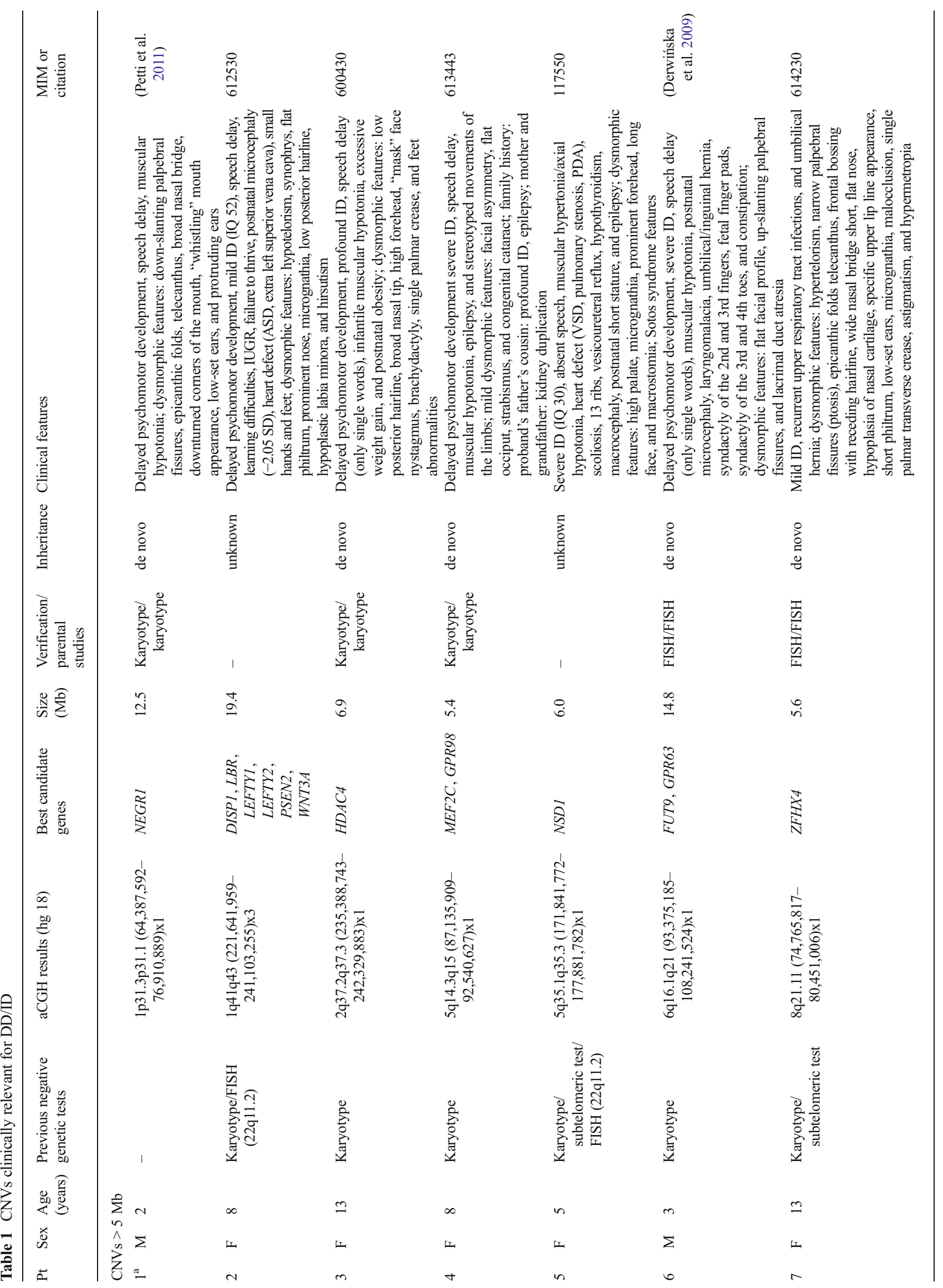




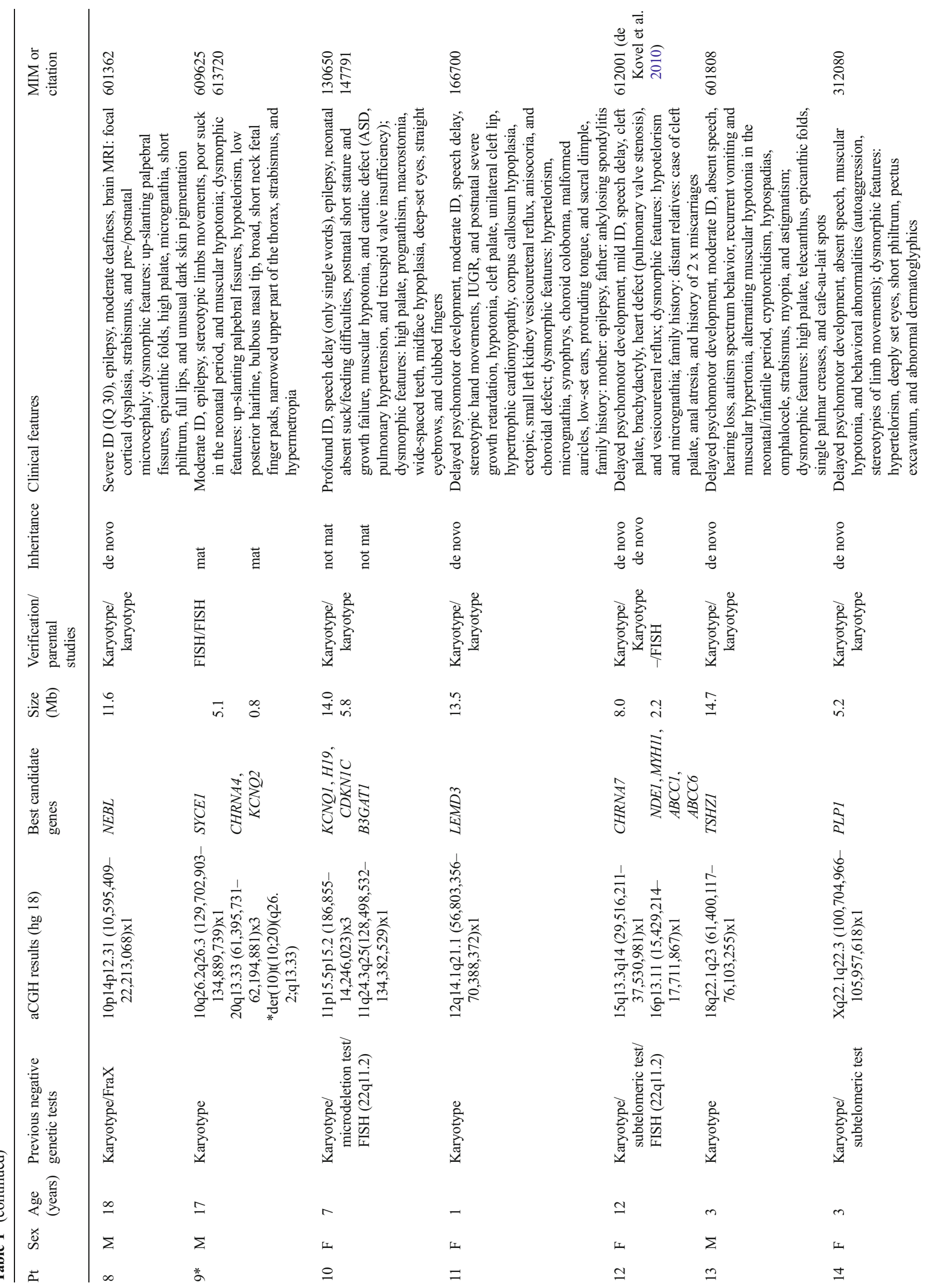




\begin{tabular}{|c|c|c|c|c|c|}
\hline \multirow[t]{2}{*}{ 这 } & 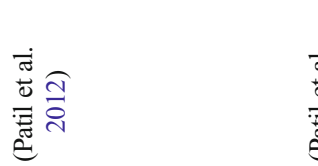 & 这 & 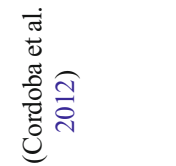 & 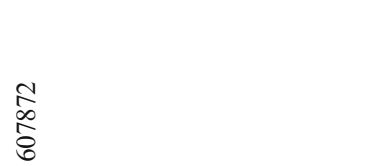 & 1 \\
\hline & 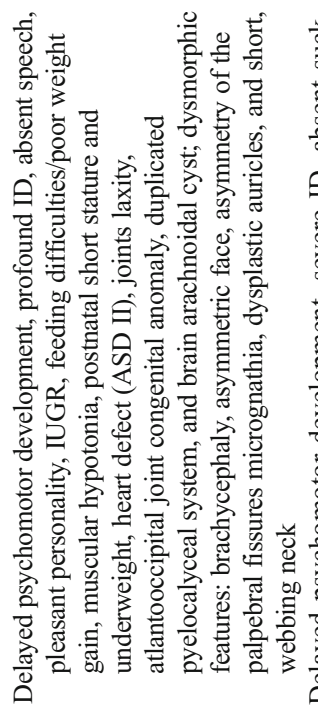 & 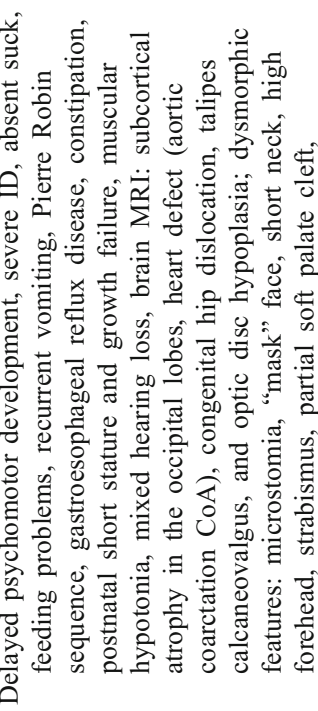 & 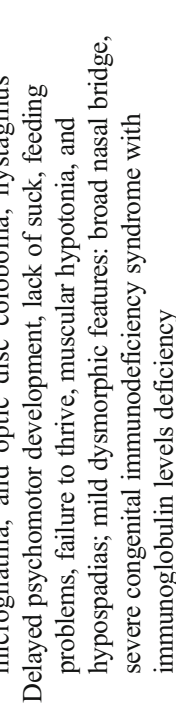 & 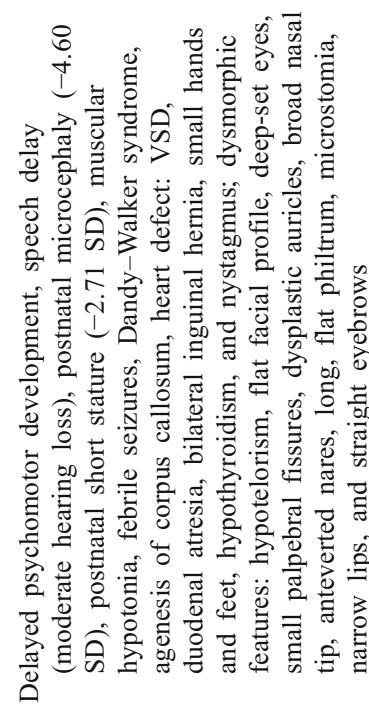 & 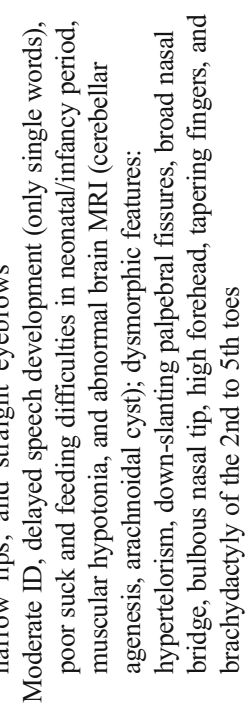 \\
\hline 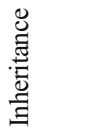 & 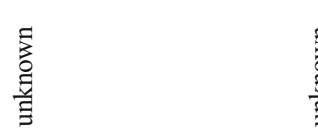 & 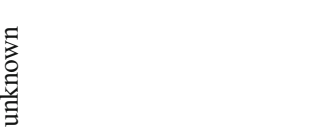 & 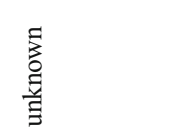 & $\begin{array}{l}\stackrel{0}{0} \\
\dot{0} \\
0\end{array}$ & $\stackrel{\overrightarrow{\tilde{\sigma}}}{2}$ \\
\hline 恶 & 咅 & 芯 & I & 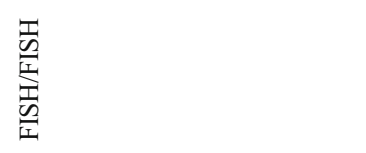 & 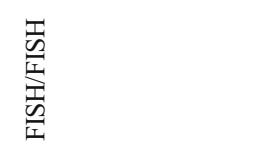 \\
\hline$\stackrel{\tilde{N}}{\tilde{E}}$ & 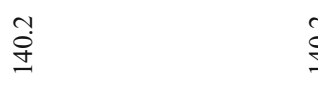 & 晏 & $\begin{array}{l}\infty \\
\infty \\
ٌ \\
ص\end{array}$ & $\stackrel{\odot}{+}$ & $\stackrel{m}{+}$ \\
\hline 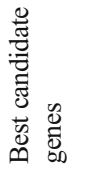 & 1 & 1 & 1 & 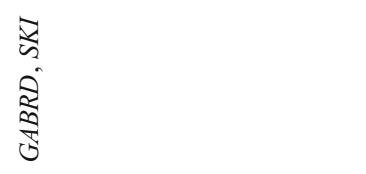 & 福 \\
\hline 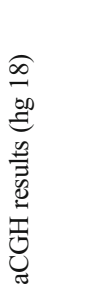 & 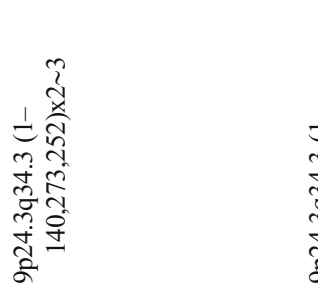 & 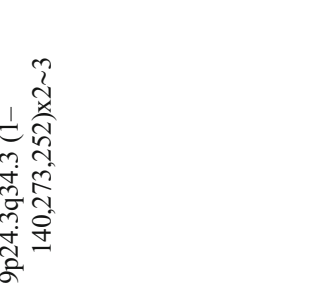 & 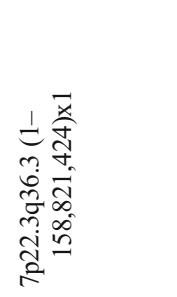 & 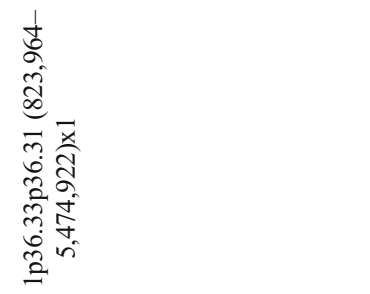 & 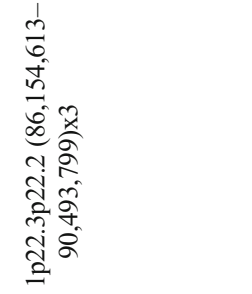 \\
\hline 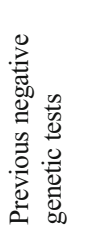 & 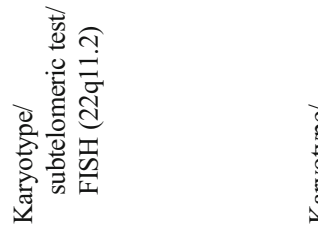 & 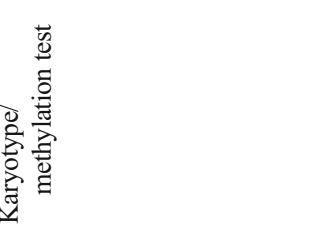 & 这 & 总 & 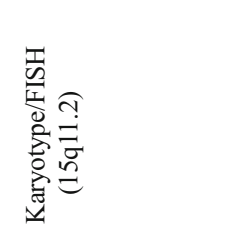 \\
\hline 品焉 & 6 & - & - & in & $\simeq$ \\
\hline 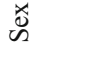 & 幽 & II & $\Sigma$ & $\Sigma$ & $\Sigma$ \\
\hline$\Xi$ & $\simeq$ & 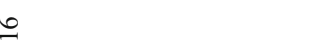 & & & $\stackrel{2}{2}$ \\
\hline
\end{tabular}




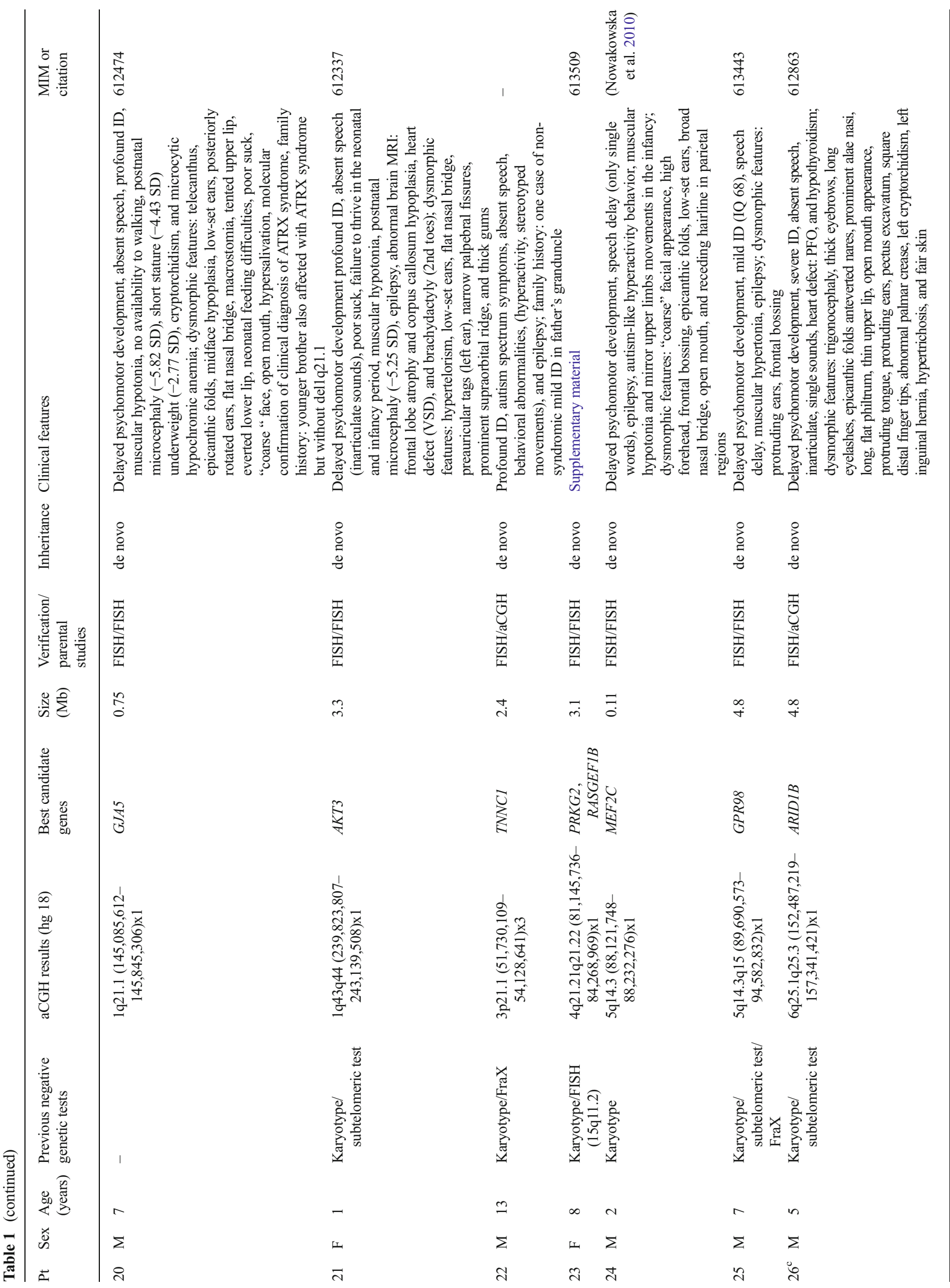




\begin{tabular}{|c|c|c|c|c|c|c|c|}
\hline \multirow[t]{2}{*}{ 茙产 } & 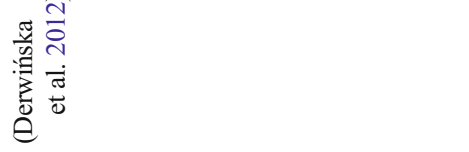 & 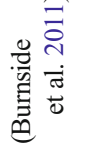 & $\begin{array}{l}\stackrel{0}{\stackrel{2}{\Xi}} \\
\stackrel{\Xi}{\Xi}\end{array}$ & $\frac{m}{\vec{b}}$ & $\begin{array}{l}\tilde{n} \\
\hat{0} \\
\frac{\pi}{b}\end{array}$ & 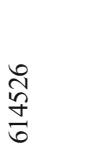 & $\frac{\frac{\pi}{d}}{6}$ \\
\hline & 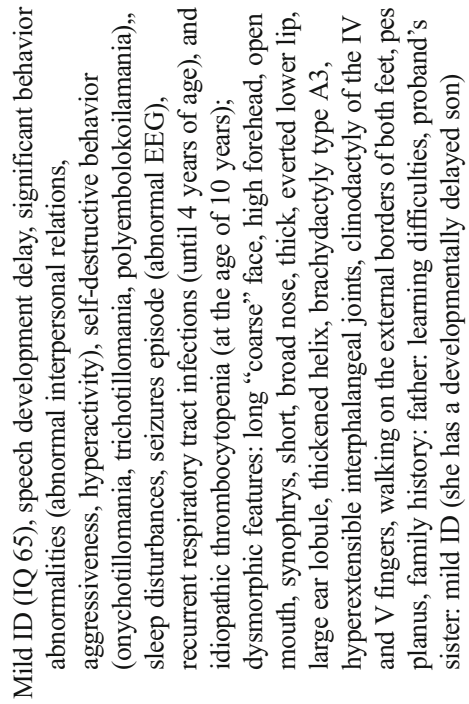 & 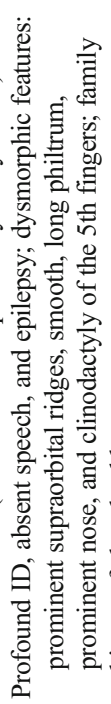 & 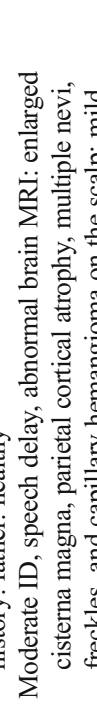 & 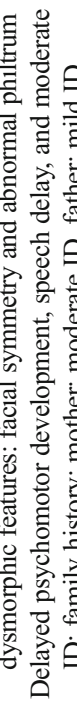 & 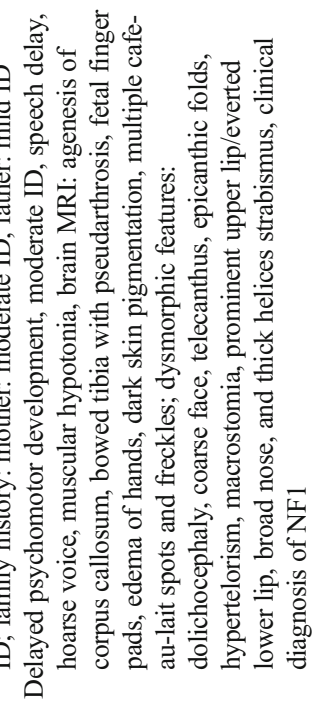 & 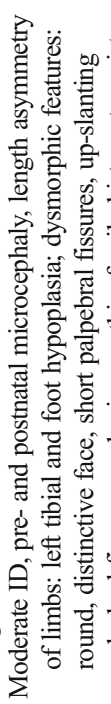 & 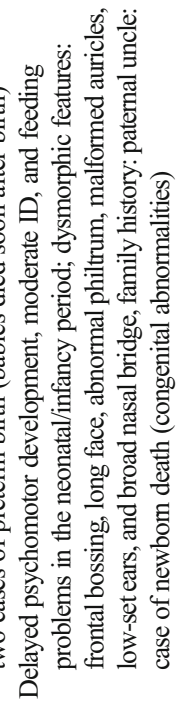 \\
\hline 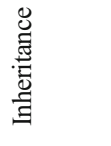 & $\begin{array}{l}\stackrel{\vec{G}}{g} \\
\stackrel{\Xi}{\circ}\end{array}$ & 苂 & $\begin{array}{l}0 \\
\stackrel{0}{0} \\
\stackrel{0}{0}\end{array}$ & 芯 & $\begin{array}{l}0 \\
\stackrel{0}{0} \\
\stackrel{0}{0} \\
0\end{array}$ & 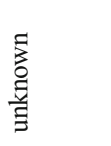 & $\begin{array}{l}0 \\
\stackrel{0}{0} \\
\stackrel{0}{0} \\
0\end{array}$ \\
\hline 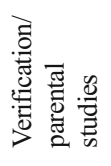 & U్: & 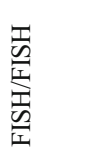 & 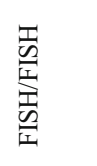 & 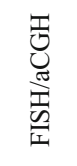 & 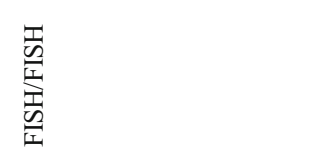 & $\frac{1}{\frac{1}{2}}$ & 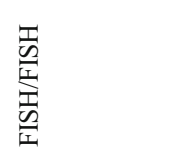 \\
\hline$\stackrel{\tilde{N}}{\hat{\Sigma}} \hat{\bar{E}}$ & $\hat{\widehat{c}}$ & $\stackrel{\text { İ }}{3}$ & $\stackrel{0}{-}$ & If & $=$ & $\exists$ & $\stackrel{t}{3}$ \\
\hline 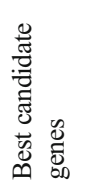 & 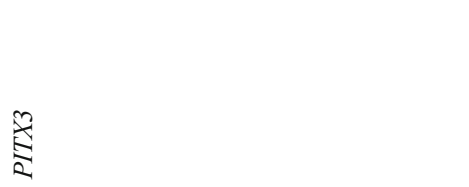 & 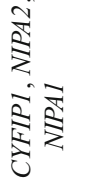 & $\begin{array}{l}\text { चี } \\
\text { सี } \\
\text { ]ี }\end{array}$ & 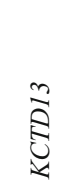 & $\stackrel{\bar{z}}{z}$ & 赵 & 空 \\
\hline 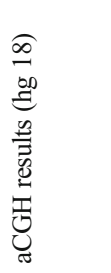 & 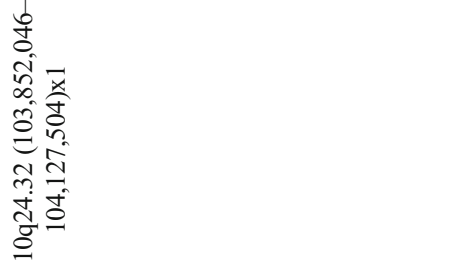 & 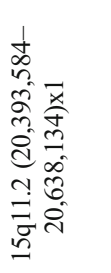 & 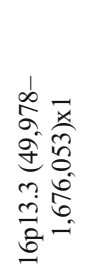 & 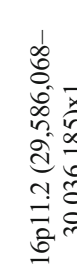 & 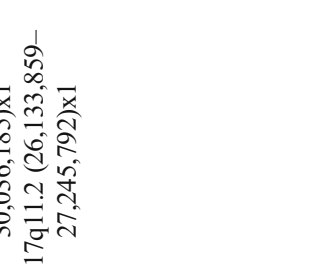 & 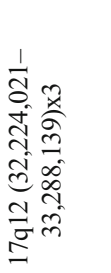 & 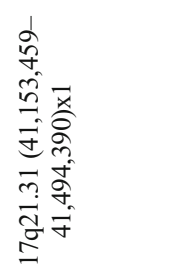 \\
\hline 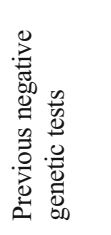 & 1 & 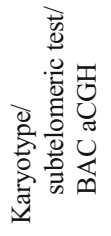 & 突 & 究 & 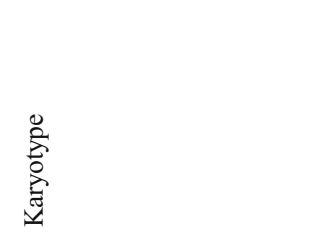 & 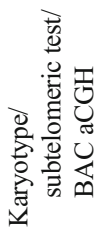 & 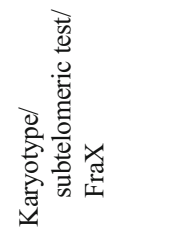 \\
\hline 总焉 & $\beth$ & r & $=$ & 6 & $\checkmark$ & $m$ & 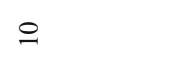 \\
\hline 蒿 & $\Sigma$ & 幽 & I & 幽 & L & I & $\Sigma$ \\
\hline$\Xi$ & $\hat{\sim}$ & $\stackrel{\infty}{\sim}$ & ते & 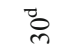 & $\bar{m}$ & ले & ल \\
\hline
\end{tabular}




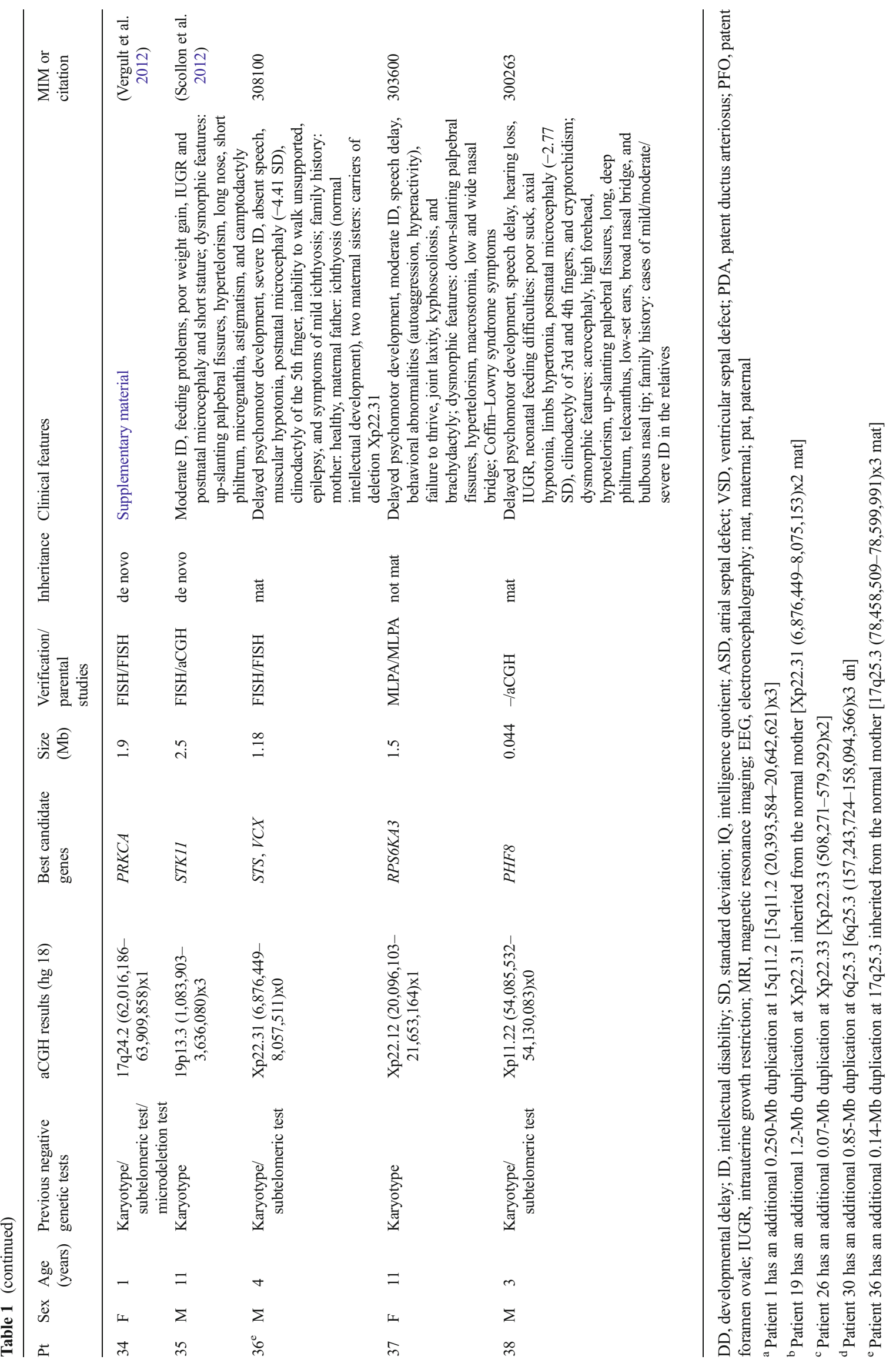




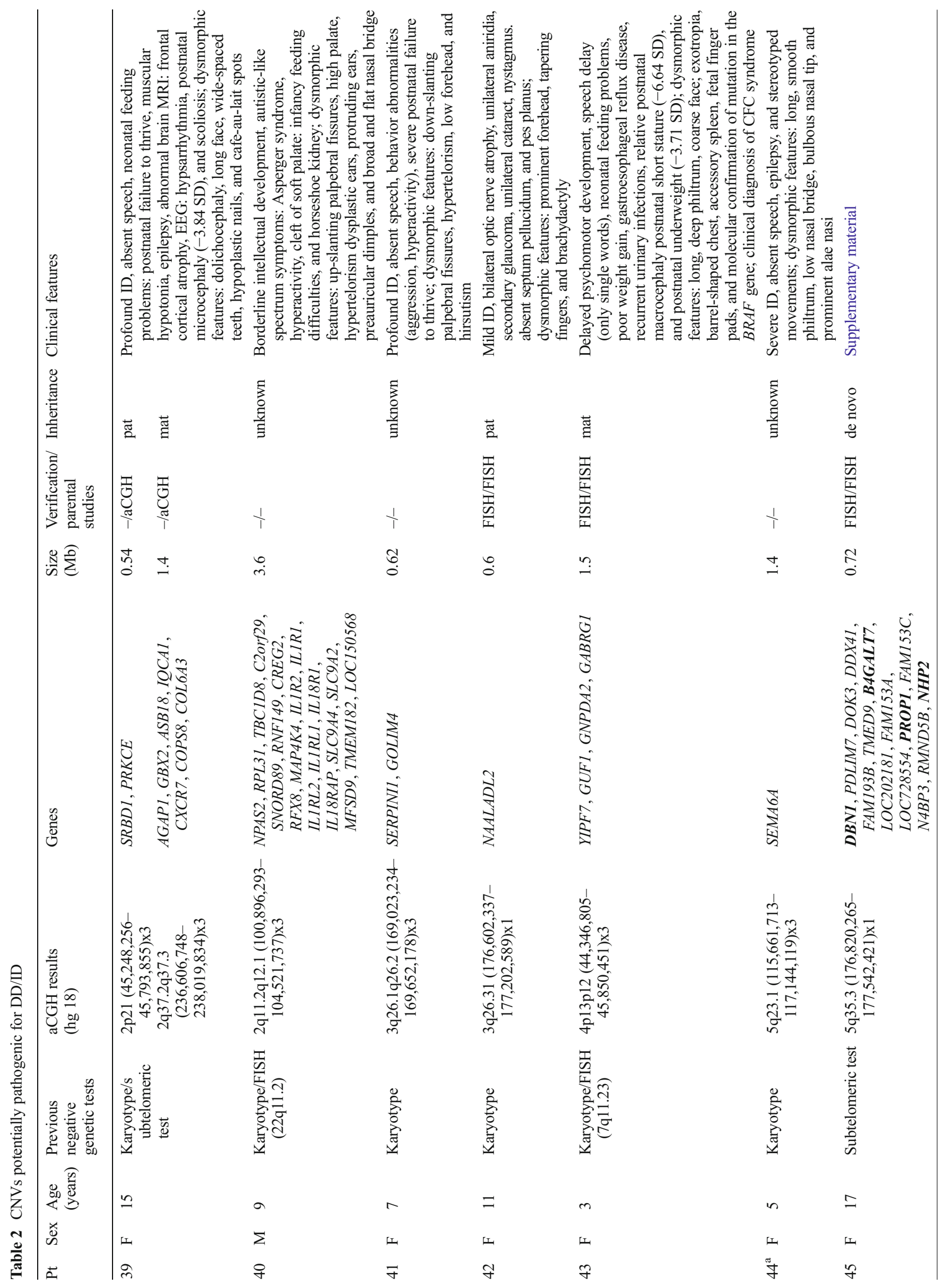




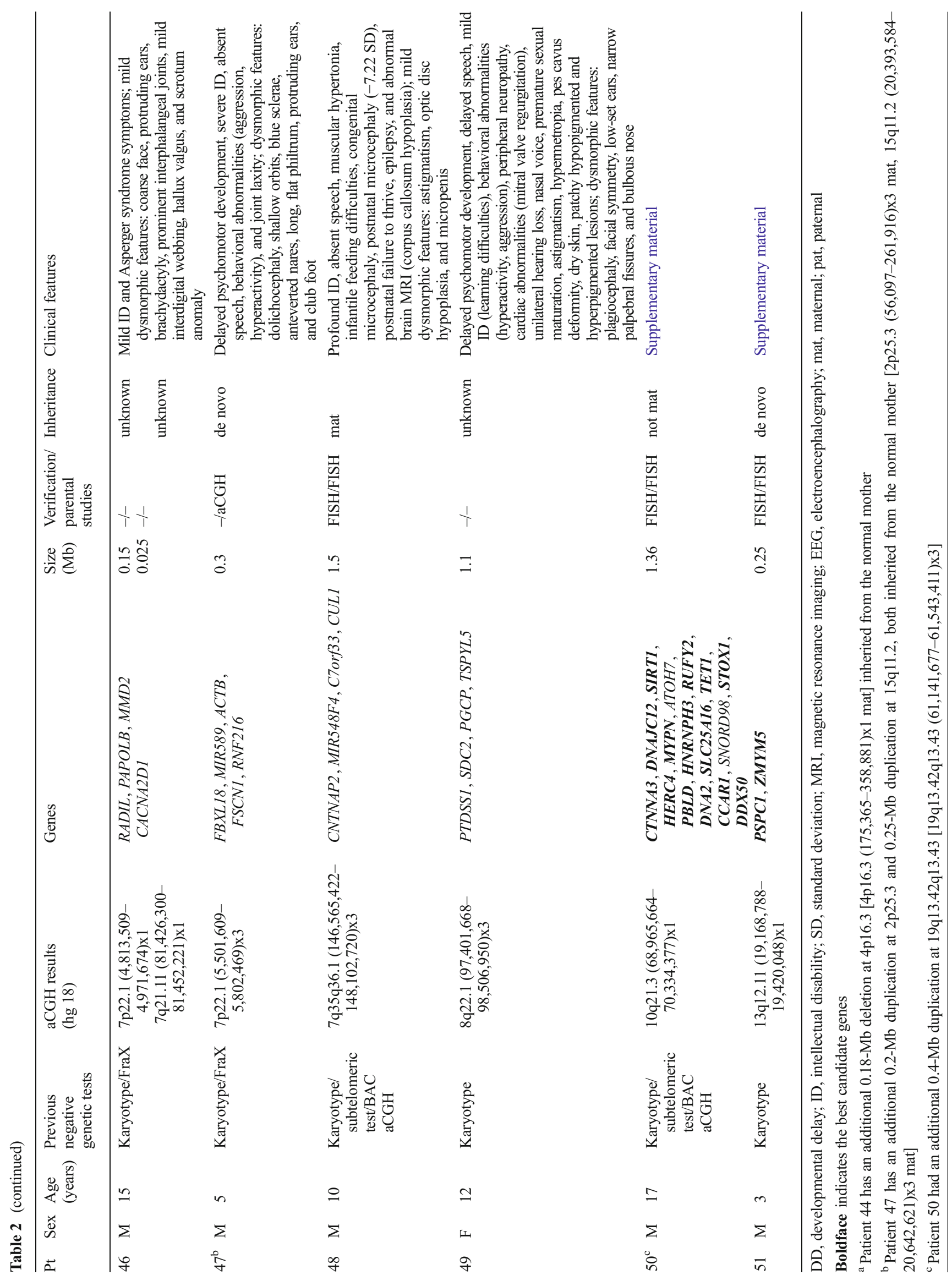


Table 3 CNVs of unknown clinical significance (likely non-pathogenic) for DD/ID

\begin{tabular}{|c|c|c|c|c|c|c|c|c|}
\hline $\mathrm{Pt}$ & Sex & $\begin{array}{l}\text { Age } \\
\text { (years) }\end{array}$ & $\begin{array}{l}\text { Previous } \\
\text { negative } \\
\text { genetic tests }\end{array}$ & aCGH results (hg 18) & $\begin{array}{l}\text { Size } \\
(\mathrm{Mb})\end{array}$ & $\begin{array}{l}\text { Verification/ } \\
\text { parental } \\
\text { studies }\end{array}$ & Inheritance & Clinical features \\
\hline
\end{tabular}

\begin{tabular}{llllcl}
\hline 52 & M & 14 & Karyotype & $\begin{array}{c}1 \mathrm{q} 21.1(144,124,745- \\
144,452,014) \times 3\end{array}$ & $0.327 \quad-/-$
\end{tabular}

unknown Moderate ID, speech delay, articulation defects, learning difficulties, and EEG abnormalities; dysmorphic features: hypertelorism, large ears, protruding ears, deep philtrum, high forehead, ptosis, prognathism, feet abnormalities, partial cutaneous syndactyly of fingers 2 and 3, and obesity since early childhood

\begin{tabular}{|c|c|c|c|c|c|c|c|c|}
\hline 53 & $\mathrm{~F}$ & 7 & Karyotype & $\begin{array}{c}5 q 14.2(82,442,763- \\
82,552,656) \times 1\end{array}$ & 0.109 & FISH/FISH & mat & $\begin{array}{l}\text { Moderate ID and behavioral abnormalities; } \\
\text { dysmorphic features: high forehead, bilateral } \\
\text { epicanthus, dysplastic, prominent and low-set } \\
\text { ears with up-lift ear lobule, broad nasal bridge, } \\
\text { thick helix, upturned nasal tip, anteverted nares, } \\
\text { prognathism, strabismus deep set eyes, and } \\
\text { clinodactyly of Vth digits }\end{array}$ \\
\hline 54 & M & 7 & Karyotype & $\begin{array}{c}7 \mathrm{p} 15.3(23,684,650- \\
23,791,776) \times 1\end{array}$ & 0.107 & FISH/FISH & pat & $\begin{array}{l}\text { Delayed psychomotor development, } \\
\text { moderate ID, speech delay, hyperactivity, } \\
\text { and learning difficulties; dysmorphic } \\
\text { features: up-slanting palpebral fissures, } \\
\text { high palate, cleft soft palate, strabismus, } \\
\text { neonatal feeding difficulties, and scoliosis; } \\
\text { suspicion of Raynaud's phenomenon }\end{array}$ \\
\hline $55^{\mathrm{a}}$ & M & 7 & $\begin{array}{l}\text { Karyotype/FISH } \\
(15 \mathrm{q} 11.2)\end{array}$ & $\begin{array}{c}9 \mathrm{p} 24.3(1,699,690- \\
3,026,335) \times 3\end{array}$ & 1.326 & $\begin{array}{l}\text { FISH/ } \\
\text { aCGH }\end{array}$ & pat & $\begin{array}{l}\text { Mildly delayed motor development, moderate } \\
\text { ID, significant delay in speech development, } \\
\text { behavioral abnormalities with aggression, } \\
\text { irritability, and short attention span, muscle } \\
\text { hypotonia, extreme obesity, hypogenitalism, } \\
\text { and ectropion of lower eyelids }\end{array}$ \\
\hline 56 & M & 1 & $\begin{array}{l}\text { Karyotype/ } \\
\text { methylation } \\
\text { test }\end{array}$ & $\begin{array}{l}\text { 11p15.4 }(4,428,038- \\
4,810,618) \times 1\end{array}$ & 0.382 & FISH/FISH & pat & $\begin{array}{l}\text { Delayed psychomotor development, muscular } \\
\text { hypotonia, epilepsy, postnatal short stature } \\
(-3.87 \mathrm{SD}) \text {, postnatal underweight } \\
\text { (-4.83 SD), Pierre Robin sequence, } \\
\text { neonatal feeding difficulties, absent suck, } \\
\text { neonatal recurrent vomiting; dysmorphic } \\
\text { features: high forehead, down-slanting } \\
\text { palpebral fissures, hypertelorism, low-set } \\
\text { ears, broad nasal bridge, strabismus, and } \\
\text { club feet; family history: father: healthy }\end{array}$ \\
\hline 57 & $\mathrm{~F}$ & 11 & $\begin{array}{l}\text { FISH (22q11.2)/ } \\
\text { BAC aCGH }\end{array}$ & $\begin{array}{l}\text { 12p13.33 }(1,738,901- \\
2,063,559) \times 3\end{array}$ & 0.324 & $\begin{array}{l}\text { FISH/ } \\
\text { aCGH }\end{array}$ & de novo & $\begin{array}{l}\text { Delayed psychomotor development, severe } \\
\text { ID, postnatal short stature, cardiac } \\
\text { abnormalities (ASD, VSD, PDA), severe } \\
\text { sensorineural deafness hypothyroidism; } \\
\text { dysmorphic features: facial asymmetry, } \\
\text { hypotelorism, ptosis, long, hooked nose, } \\
\text { cleft palate, micrognathia, neck webbing, } \\
\text { radioulnar synostosis, myopia, accessory } \\
\text { spleen, family history: } 1 \times \text { stillbirth without } \\
\text { congenital malformations, } 1 \times \text { miscarriage } \\
\text { (first trimester of gestation) }\end{array}$ \\
\hline 58 & M & 2 & Karyotype & $\begin{array}{l}\text { 12q21.1 }(70,966,697- \\
71,222,382) \times 1\end{array}$ & 0.255 & FISH/FISH & pat & $\begin{array}{l}\text { Delayed psychomotor development, absent } \\
\text { speech (inarticulate, single sounds), in the } \\
\text { neonatal/infantile period of life: limbs } \\
\text { hypertonia/axial hypotonia, seizures, } \\
\text { pre-/postnatal microcephaly: (-5.65 SD), } \\
\text { epilepsy, brain MRI: suspicion of septo-optic } \\
\text { dysplasia, polymicrogyria, schizencephaly, } \\
\text { and subependymal heterotopy; dysmorphic } \\
\text { features: down-slanting palpebral fissures, }\end{array}$ \\
\hline
\end{tabular}


Table 3 (continued)

\begin{tabular}{|c|c|c|c|c|c|c|}
\hline $\mathrm{Pt}$ & Sex & $\begin{array}{l}\text { Age } \\
\text { (years) }\end{array}$ & $\begin{array}{l}\text { Previous } \\
\text { negative } \\
\text { genetic tests }\end{array}$ & aCGH results (hg 18) & $\begin{array}{l}\text { Size } \\
(\mathrm{Mb})\end{array}$ & $\begin{array}{l}\text { Verification/ Inheritance Clinical features } \\
\text { parental } \\
\text { studies }\end{array}$ \\
\hline
\end{tabular}

\begin{tabular}{|c|c|c|c|c|c|c|c|c|}
\hline & & & & & & & & $\begin{array}{l}\text { hypotelorism, EEG abnormalities, } \\
\text { genitourinary abnormalities: extrarenal pelvis } \\
\text { (left kidney) and optic disc hypoplasia }\end{array}$ \\
\hline 59 & M & 8 & $\begin{array}{l}\text { Karyotype/ } \\
\text { subtelomeric } \\
\text { test }\end{array}$ & $\begin{array}{l}13 \mathrm{q} 32.1 \mathrm{q} 32.2 \\
\quad(96,900,637- \\
97,686,612) \times 3\end{array}$ & 0.785 & $\begin{array}{l}\text { FISH/ } \\
\text { aCGH }\end{array}$ & mat & $\begin{array}{l}\text { Delayed psychomotor development, profound } \\
\text { ID, absent speech, neonatal and infantile } \\
\text { feeding problems, muscular limbs hypertonia, } \\
\text { seizures, drug-resistant epilepsy, pre- and } \\
\text { postnatal microcephaly, stereotyped } \\
\text { movements; dysmorphic features } \\
\text { brachycephaly, micrognathia, protruding ears, } \\
\text { open mouth appearance, full cheeks, } \\
\text { abnormal brain MRI: temporal cortical } \\
\text { atrophy (right), scoliosis, cryptorchidism, } \\
\text { nystagmus, hematologic abnormalities, and } \\
\text { unexplained transient highly elevated } \\
\text { leukocytosis (30,000/ml); family history: } \\
\text { proband's sister: very similar clinical } \\
\text { symptoms suggestive for unexplained } \\
\text { encephalopathy with drug-resistant epilepsy }\end{array}$ \\
\hline 60 & M & 15 & Karyotype & $\begin{array}{c}15 \mathrm{q} 11.2(19,924,765- \\
20,642,621) \times 3\end{array}$ & 0.717 & $-1-$ & unknown & $\begin{array}{l}\text { Delayed psychomotor development, profound } \\
\text { ID, absent speech, stereotyped movements, } \\
\text { neonatal/infantile feeding difficulties, } \\
\text { poor suck, poor weight gain, postnatal } \\
\text { failure to thrive, pectus excavatum, } \\
\text { chondro-osseous exostoses on the } \\
\text { lower limbs, strabismus, and secondary } \\
\text { osteoporosis (hypercalciuria) }\end{array}$ \\
\hline 61 & M & 10 & Karyotype & $\begin{array}{l}15 \mathrm{q} 11.2(20,006,102- \\
20,642,621) \times 3\end{array}$ & 0.636 & $-1-$ & unknown & $\begin{array}{l}\text { Delayed psychomotor development, moderate } \\
\text { ID, speech delay, articulation defect, learning } \\
\text { difficulties, vertebral and rib anomalies, short } \\
\text { neck, postnatal short stature (relatively short } \\
\text { lower limbs), postnatal underweight, heart } \\
\text { defect (ASD, VSD), and strabismus; family } \\
\text { history: } 1 \times \text { miscarriage in the mothers' } \\
\text { gestational history; features of Jarcho-Levin- } \\
\text { like syndrome }\end{array}$ \\
\hline 62 & M & 6 & $\begin{array}{l}\text { Subtelomeric } \\
\text { test }\end{array}$ & $\begin{array}{c}15 \mathrm{q} 21.3(54,693,214- \\
54,832,701) \times 1\end{array}$ & 0.139 & FISH/FISH & mat & $\begin{array}{l}\text { Delayed psychomotor development, moderate } \\
\text { ID, neonatal/infantile feeding difficulties, } \\
\text { joints laxity, pectus excavatus, strabismus, } \\
\text { and heart defect (VSD); dysmorphic } \\
\text { features: Kabuki-like makeup syndrome, } \\
\text { ectropion of eyelids, wide palpebral fissures, } \\
\text { epicanthic folds, prominent ears, flat } \\
\text { philtrum, and fetal finger pads }\end{array}$ \\
\hline 63 & F & 14 & Karyotype/FraX & $\begin{array}{l}\text { 16q23.1 }(77,445,915- \\
78,190,209) \times 3\end{array}$ & 0.744 & $-/ \mathrm{aCGH}$ & not mat & $\begin{array}{l}\text { Mild/moderate ID, mild dysmorphic features, } \\
\text { hypertelorism, high forehead transverse } \\
\text { palmar crease, and low posterior hairline }\end{array}$ \\
\hline 64 & F & 6 & $\begin{array}{l}\text { Karyotype/BAC } \\
\text { aCGH/ } \\
\text { methylation } \\
\text { test }\end{array}$ & $\begin{array}{l}22 \mathrm{q} 11.21 \mathrm{q} 11.22 \\
\quad(20,269,922- \\
21,393,710) \times 3\end{array}$ & 1.123 & $-1-$ & unknown & $\begin{array}{l}\text { Severe ID, absent speech, stereotyped } \\
\text { movements, breath holding, postnatal } \\
\text { microcephaly, inability to walk } \\
\text { independently, rash of upper limbs; } \\
\text { dysmorphic features, short philtrum, uplift } \\
\text { earlobes, thick and sparse eyebrows, flat } \\
\text { facial profile, protruding tongue, exotropia, } \\
\text { and gum hypertrophy; family history: } 1 \times \\
\text { miscarriage; clinical diagnosis of Rett } \\
\text { syndrome, molecular confirmation of } \\
M E C P 2 \text { mutation }\end{array}$ \\
\hline
\end{tabular}


Table 3 (continued)

\begin{tabular}{|c|c|c|c|c|c|c|c|c|}
\hline $\mathrm{Pt}$ & Sex & $\begin{array}{l}\text { Age } \\
\text { (years) }\end{array}$ & $\begin{array}{l}\text { Previous } \\
\text { negative } \\
\text { genetic tests }\end{array}$ & aCGH results (hg 18) & $\begin{array}{l}\text { Size } \\
(\mathrm{Mb})\end{array}$ & $\begin{array}{l}\text { Verification/ } \\
\text { parental } \\
\text { studies }\end{array}$ & Inheritance & Clinical features \\
\hline 65 & M & 11 & $\begin{array}{l}\text { Karyotype/ } \\
\text { subtelomeric } \\
\text { test }\end{array}$ & $\begin{array}{l}\text { Xp22.33 }(263,265- \\
690,981) \times 3\end{array}$ & 0.427 & $-/-$ & unknown & $\begin{array}{l}\text { Mild ID, speech delay, Postnatal microcephaly, } \\
\text { abnormal brain MRI: arachnoid cyst and } \\
\text { cerebellar vermis hypoplasia, EEG } \\
\text { abnormalities, heart defect: tetralogy of } \\
\text { Fallot (TOF), unilateral inguinal hernia, and } \\
\text { vesicoureteral reflux; dysmorphic features: } \\
\text { hypertelorism, telecanthus, short philtrum, } \\
\text { downturned corners of the mouth, and thin } \\
\text { lips }\end{array}$ \\
\hline 66 & $\mathrm{~F}$ & 4 & - & $\begin{array}{r}\text { Xp22 }(502,226- \\
1,744,236) \times 3\end{array}$ & 1.242 & $-1-$ & unknown & $\begin{array}{l}\text { Delayed psychomotor development, severe } \\
\text { ID, speech delay (only single words), and } \\
\text { autistic spectrum behavior; dysmorphic } \\
\text { features: almond-shaped eyes, short } \\
\text { philtrum, downturned corners of the } \\
\text { mouth, prominent nasal tip, and proximal } \\
\text { placement of the thumbs; family history: } \\
1 \times \text { miscarriage in the mother }\end{array}$ \\
\hline 67 & $\mathrm{~F}$ & 18 & $\begin{array}{l}\text { Karyotype/ } \\
\text { subtelomeric } \\
\text { test/FraX/ } \\
\text { FISH(1p36) }\end{array}$ & $\begin{array}{c}\text { Xq27.3 }(142,571,903- \\
142,766,693) \times 3\end{array}$ & 0.194 & $-/ \mathrm{aCGH}$ & not mat & $\begin{array}{l}\text { Profound ID and absent speech (only single } \\
\text { words); dysmorphic features: deep-set eyes, } \\
\text { straight supraorbital ridges, postnatal } \\
\text { microcephaly, behavior abnormalities: } \\
\text { aggression, autoaggression, stereotyped } \\
\text { movements, obesity, and small hands and } \\
\text { feet; family history: mother: mild ID }\end{array}$ \\
\hline 68 & $\mathrm{~F}$ & 2 & $\begin{array}{l}\text { Karyotype/ } \\
\text { subtelomeric } \\
\text { test/HR-CGH }\end{array}$ & $\begin{array}{c}\text { Xq28 }(151,572,279- \\
151,788,528) \times 3\end{array}$ & 0.216 & $-/ \mathrm{aCGH}$ & pat & $\begin{array}{l}\text { Delayed psychomotor development, speech } \\
\text { delay (only single words), relative postnatal } \\
\text { microcephaly }(-2.05 \mathrm{SD}) \text {, feeding } \\
\text { difficulties, absent suck, distal } \\
\text { arthrogryposis, (dimples over the } \\
\text { interphalangeal joints), and premature } \\
\text { thelarche; dysmorphic features: up-slanting } \\
\text { palpebral fissures, long philtrum, } \\
\text { downturned corners of the mouth, metopic } \\
\text { ridge, and strabismus }\end{array}$ \\
\hline 69 & M & 15 & $\begin{array}{l}\text { Karyotype/ } \\
\text { subtelomeric } \\
\text { test/FraX }\end{array}$ & $\begin{array}{c}\text { Xq28 }(152,641,149- \\
152,697,974) \times 3\end{array}$ & 0.056 & $-1-$ & unknown & $\begin{array}{l}\text { Profound ID, absent speech, behavioral } \\
\text { abnormalities (hyperactivity, aggression), } \\
\text { autism spectrum symptoms, muscular } \\
\text { hypotonia, microcephaly, epilepsy, } \\
\text { brachydactyly, alopecia areata, thin skin } \\
\text { with hyperpigmentation in the perioral and } \\
\text { periorbital regions; family history: stillbirth } \\
\text { newborn from the mothers' first pregnancy } \\
\text { died after birth (IUGR without congenital } \\
\text { malformations) and } 2 \times \text { miscarriages at the } \\
\text { first trimester of the mother's pregnancies }\end{array}$ \\
\hline
\end{tabular}

DD, developmental delay; ID, intellectual disability; SD, standard deviation; ASD, atrial septal defect; VSD, ventricular septal defect; PDA, patent ductus arteriosus; IUGR, intrauterine growth restriction; MRI, magnetic resonance imaging; EEG, electroencephalography; mat, maternal; pat, paternal The total number of CNVs of unknown clinical significance includes CNVs in Table 3 and the additional changes listed under Tables 1, 2, and 3

${ }^{a}$ Patient 55 with a karyotype 46,XY,t(2;11)(q21;q23) has an additional $0.754-\mathrm{Mb}$ deletion at 2q22.11 inherited from the normal mother [2q22.1 $(138,045,798-138,799,860) \times 1$ mat]

belongs to the zinc finger MYM-type family and probably has molecular functions, such as metal ion binding or zinc ion binding (Pastorcic and Das 2007). Although the phenotypegenotype correlation remains unclear and one smaller deletion within this region was reported in the control group (DGV; nsv455826) (Itsara et al. 2009), we propose that the identified de novo $13 q 12.11$ deletion could contribute to the clinical features observed in our patient. 
Table 4 Summary of the studied cohort of 256 patients with DD/ID

\begin{tabular}{|c|c|c|c|c|c|c|}
\hline \multirow[t]{2}{*}{ Patients with } & \multirow{2}{*}{$\begin{array}{l}\text { Total number } \\
\text { (256) }\end{array}$} & \multirow{2}{*}{$\begin{array}{l}\text { Number of patients } \\
\text { with CNVs (69) }\end{array}$} & \multicolumn{3}{|c|}{ Reported CNVs } & \multirow{2}{*}{$\begin{array}{l}\text { Number of patients } \\
\text { with two CNVs }\end{array}$} \\
\hline & & & $\begin{array}{l}\text { Pathogenic } \\
\text { (41) }\end{array}$ & $\begin{array}{l}\text { Potentially } \\
\text { pathogenic (15) }\end{array}$ & $\begin{array}{l}\text { Variants of unknown } \\
\text { significance }(28)\end{array}$ & \\
\hline DD (age $1-6$ years) & 74 & $17(22.9 \%)$ & $12(16.2 \%)$ & 2 & 4 & 1 \\
\hline Mild ID & 32 & $11(34.3 \%)$ & $6(18.7 \%)$ & 5 & 2 & 2 \\
\hline Age $<12$ years & 22 & 7 & 4 & 3 & 1 & - \\
\hline Age $13-18$ years & 8 & 4 & 2 & 2 & 1 & - \\
\hline Age $>18$ years & 2 & - & - & - & - & - \\
\hline Moderate ID & 57 & $18(31.5 \%)$ & $11(19.2 \%)$ & 2 & 10 & 5 \\
\hline Age $<12$ years & 35 & 13 & 8 & - & 8 & - \\
\hline Age $13-18$ years & 20 & 4 & 2 & 2 & 2 & - \\
\hline Age $>18$ years & 2 & 1 & 1 & - & - & - \\
\hline Severe ID & 32 & $10(31.2 \%)$ & $5(15.6 \%)$ & 2 & 8 & 4 \\
\hline Age $<12$ years & 25 & 9 & 4 & 2 & 8 & - \\
\hline Age $13-18$ years & 7 & 1 & 1 & - & - & - \\
\hline Age $>18$ years & - & - & - & - & - & - \\
\hline Profound ID & 61 & $13(21.3 \%)$ & $7(11.4 \%)$ & 4 & 4 & 2 \\
\hline Age $<12$ years & 35 & 7 & 5 & 2 & 1 & - \\
\hline Age $13-18$ years & 19 & 6 & 2 & 2 & 3 & - \\
\hline Age $>18$ years & 7 & - & - & - & - & - \\
\hline
\end{tabular}

In a girl (pt 45) with an atypical autism spectrum disorder and moderate mental impairment with absent expressive speech development, we detected a de novo $\sim 720-\mathrm{kb}$ deletion at $5 \mathrm{q} 35.3$ that partially overlaps a common-sized $\sim 1.9-\mathrm{Mb}$ recurrent deletion found in patients with Sotos syndrome (MIM 117550) but leaves the dosage-sensitive NSD1 gene (set domain protein 1; MIM 606681) intact (Fig. 1b, e). Rauch et al. (2003) described a novel 5q35.3 subtelomeric deletion distally adjacent to the Sotos common deletion region, and characterized by pronounced muscular hypotonia, postnatal short stature, and bell-shaped thorax with pectus carinatum. The deletion identified in our patient harbors 15 genes, including DBN1, B4GALT7, PROP1, and NHP2.DBN1 (drebrin E; MIM 126660) is a cytoplasmic actin-binding protein thought to play a role in neuronal growth and dendritic spine formation. It is a member of the drebrin family of proteins that are developmentally regulated in the brain. Shim and Lubec (2002) and Dun and Chilton (2010) suggested that a decreased amount of drebrin may lead to loss of spine plasticity and impaired dendritic arborization, which may underlie cognitive dysfunction. PROP1 (paired-like homeodomain transcription factor; MIM 601538) has both DNA-binding and transcriptional activation ability. Its expression leads to ontogenesis of pituitary gonadotropes, as well as somatotropes, lactotropes, and caudomedial thyrotropes. Heterozygous mutations in PROP1 have been reported in patients with pituitary hormone deficiency-2 (CPHD2; MIM 262600). Homozygous mutations of B4GALT7 (galactosyltransferase I; MIM 604327) have been described in patients with the progeroid form of EhlersDanlos syndrome (MIM 130070) characterized by an aged appearance, DD, short stature, craniofacial disproportion, generalized osteopenia, defective wound healing, hypermobile joints, hypotonic muscles, and loose but elastic skin (Okajima et al. 1999). Lastly, homozygous or compound heterozygous mutations in the NHP2 gene (nucleolar protein family A, member 2; MIM 606470) have been found in individuals with autosomal recessive dyskeratosis congenita2 (DKCB2; MIM 613987). Given that the 5q35.3 deletion in our patient arose de novo and the fact that the deleted genes are associated with neurodevelopmental disorders and play a role in neuronal processes, we suggest that the identified $\mathrm{CNV}$ is potentially pathogenic for the described clinical features.

In patient 50 with short stature, moderate ID, and history of Hirschsprung disease and congenital heart defect, we found a rare $1.4-\mathrm{Mb}$ deletion in 10q21.3 encompassing 16 genes (Fig. 1c, f) and a small $\sim 400-\mathrm{kb}$ duplication in 19q13.42q13.43. Deletions in 10q21.3 have never been reported in patients with $\mathrm{DD} / \mathrm{ID}$, thus, it is challenging to assess its clinical pathogenicity. However, the size and gene-rich content suggest that the 10q21.3 deletion could be pathogenic. Analysis of the maternal sample revealed normal result; unfortunately, the paternal sample was unavailable. Five genes, including SIRT1 (sirtuin 1; MIM 604479), DNA2 (DNA replication helicase 2; MIM 601810), TET1 (Tet oncogene family, member 1; MIM 607790), CCAR1 (cell division cycle and apoptosis regulator 1; MIM 612569), and DDX50 


\section{a $13 q 12.11$ region}
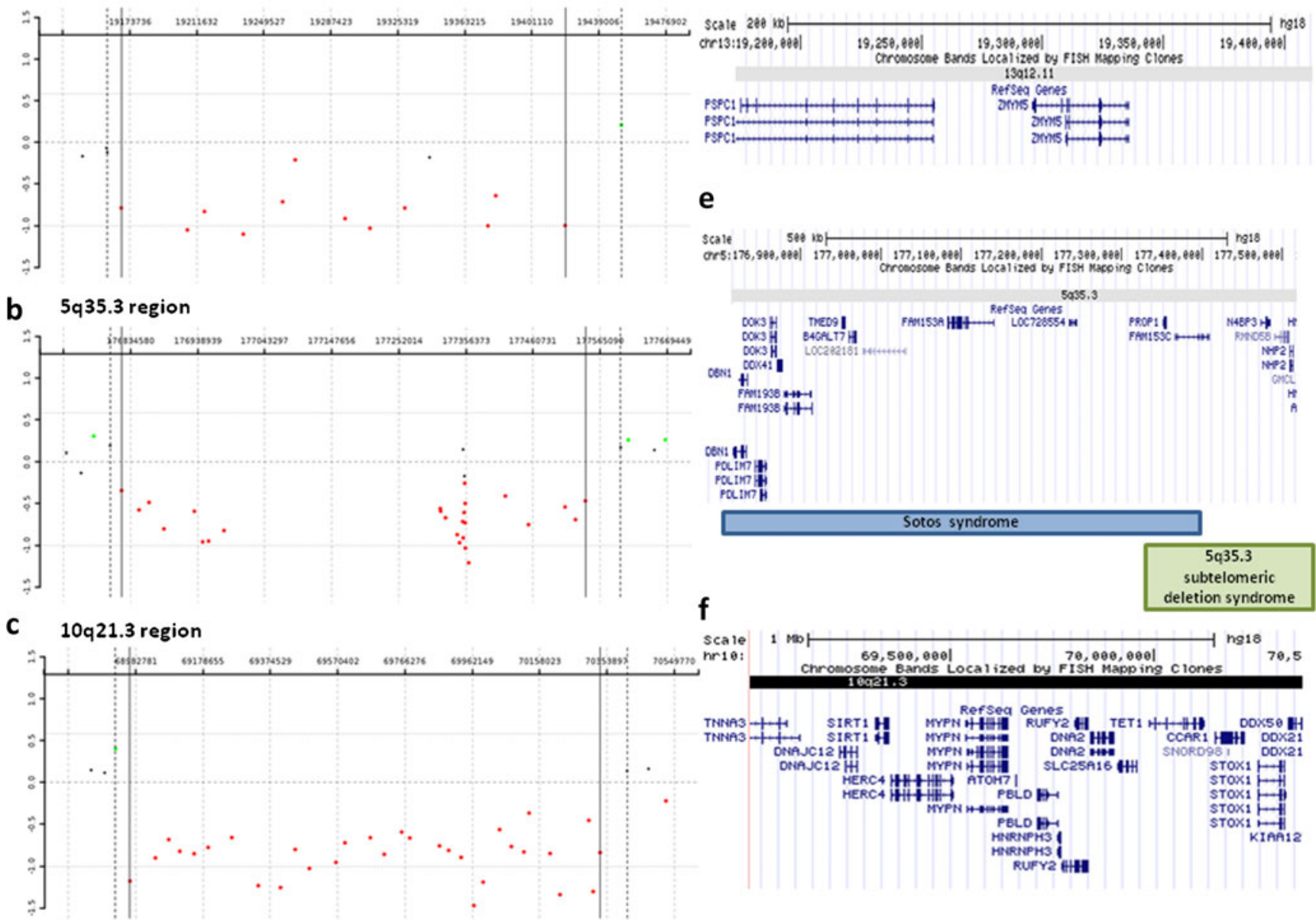

Fig. 1 Results of array CGH analyses: a patient 51, showing a de novo $\sim 250-\mathrm{kb}$ deletion in 13q12.11; b patient 45 , demonstrating a de novo $\sim 720-\mathrm{kb}$ deletion at $5 \mathrm{q} 35.3$; and $\mathbf{c}$ patient 50 , showing a rare $\sim 1.4-\mathrm{Mb}$ deletion in 10q21.3. The red dots denote the deleted region. Gene content

in the deleted region on: $\mathbf{d}$ chromosome 13q12.11; e chromosome $5 \mathrm{q} 35.3$ (compared with the critical region of the Sotos syndrome and $5 \mathrm{q} 35.3$ subtelomeric deletion syndrome); and $\mathbf{f}$ chromosome 10q21.3 (UCSC genome browser, http://genome.ucsc.edu/)

(dead/h box 50; MIM 610373) represent dosage-sensitive genes (Huang et al. 2010); however, to date, no phenotype associated with haploinsufficiency of any of these genes has been reported. In addition, SLC25A16 (solute carrier family 25, member 16; MIM 139080) encodes a protein that is localized in the inner membrane and facilitates the rapid transport and exchange of molecules between the cytosol and the mitochondrial matrix space. This gene was proposed to play a role in Graves disease (MIM 275000). Of note, other SLC family genes, SLC9A9 (MIM 608396), SLC6A4 (MIM 182138), and SLC25A12 (MIM 603667), are causative for autism. Other genes mapping in the deleted region, including DNAJC12 (MIM 606060), HERC4 (MIM 609248), PBLD (MIM 612189), HNRNPH3 (MIM 602324), RUFY2 (MIM 610328), STOX1 (MIM 609397), and CTNNA3 (MIM 607667), have been linked to late-onset Alzheimer's disease (AD6; MIM 605526). CTNNA3, encoding the alpha-3 catenin and likely responsible for the formation of stretch-resistant cell-cell adhesion complexes (Weiss et al. 2009), has been associated with late-onset Alzheimer's disease in females (Miyashita et al. 2007). Bradley et al. (2010) suggested that, if CTNNA3 is involved in Alzheimer's disease, it is not through a loss-of-function mechanism. Moreover, we suggest that CTNNA3 and/or MYPN (myopalladin, MIM 608517), deleted 10q21 region, can be responsible for the congenital heart defect observed in our patient. CTNNA3 has been considered as a candidate for the form of dilated cardiomyopathy linked to 10q21q23 (CMD1C; 601493) because of its high expression in the heart. MYPN is a component of the sarcomere that tethers nebulette in cardiac muscle to alphaactinin and may play signaling roles in targeting and orienting nebulin to the $\mathrm{Z}$ line during sarcomere assembly. The associated $\sim 400-\mathrm{kb}$ duplication in $19 \mathrm{q} 13.42 \mathrm{q} 13.43$ harbors seven genes (NLRP8; MIM 609659, NLRP5; MIM 609658, ZNF787, ZNF444; MIM 607874, GALP; MIM 611178, ZSCAN5B, and ZSCAN5A), none of which represent a 
dosage-sensitive gene. Therefore, we classified this duplication as a variant of unknown clinical significance.

Among our $41 \mathrm{CNVs}$ known as being pathogenic for DD/ ID, we elected to discuss two deletions, 4q21 and 17q24.2, which have been described recently as responsible for microdeletion syndromes associated with DD/ID. Moreover, in those patients (pt 23 and pt 34), previously performed genetic tests were negative (Table 1), which demonstrates the usefulness of array CGH as a great tool for identifying the etiology of idiopathic DD/ID.

A de novo 3.1-Mb deletion at 4q21.21q21.22 identified in patient 23 overlaps CNVs reported in the literature (Harada et al. 2002; Friedman et al. 2006; Bonnet et al. 2010; Lipska et al. 2011). Bonnet et al. (2010) defined a 1.37-Mb critical region containing five genes, PRKG2 (MIM 601591), RASGEFIB (MIM 614532), HNRNPD (MIM 601324), HNRPDL (MIM 607137), and ENOPH1, and proposed that $P R K G 2$ and $R A S G E F 1 B$ are the best candidate genes responsible for the 4q21 deletion syndrome (MIM 613509) characterized by severe ID, lack of speech, hypotonia, significant growth restriction, and distinctive facial features. Our patient enables a better delineation of a novel $4 \mathrm{q} 21$ microdeletion syndrome and further supports the proposed contribution of haploinsufficiency of PRKG2 and RASGEF1B.

We also identified a de novo $\sim 1.9-\mathrm{Mb}$ deletion at $17 \mathrm{q} 24.2$ (pt 34), harboring the smallest region of overlap of four deletions recently reported by Vergult et al. (2012). The shared clinical features include ID, speech delay, truncal obesity, and similar facial gestalt. The $\sim 713-\mathrm{kb}$ critical region contains five genes, including PRKCA (MIM 176960), a cluster of three $C A C N G$ genes encoding the gamma subunit of a voltagedependent calcium channel, CACNG5 (MIM 606405), CACNG4 (MIM 606404), CACNG1 (MIM 114209), and HELZ (MIM 606699). The PRKCA gene encodes the serine- and threonine-specific protein kinase $\mathrm{C}$ alpha that plays an important role in many different cellular processes and is the most important candidate gene for many of the observed clinical features. Investigations of more patients with $17 q 24.2$ deletions would strengthen the genotype-phenotype correlation in this newly reported microdeletion syndrome.

Moreover, in two patients (pt 15 and pt 16) with normal standard cytogenetic results, we detected chromosomal aneuploidy in the form of a mosaic trisomy 9 using array CGH. In both cases, chromosomal mosaicism was confirmed by retrospective GTG banding analysis that revealed low-level mosaicism for trisomy 9 of $12.5 \%$ and $8 \%$, respectively. Additionally, in patient 17 with DD and combined immunodeficiency and normal karyotype, we found a monosomy of chromosome 7 in his peripheral blood leukocytes. Chromosome 7 monosomy and $7 \mathrm{q}$ deletion have been frequently found in patients with myelodysplastic syndrome (MDS). MDSs are a heterogeneous group of stem cell disorders characterized by ineffective hematopoiesis, dysplastic changes in bone marrow and peripheral blood, and the risk of transformation to acute myeloid leukemia (AML) (Cordoba et al. 2012). We suggest that the identified aberration is responsible for the phenotypic abnormalities observed in our patient.

Additionally, our analyses of the DD/ID cohort revealed patients with more than one clinically relevant CNVs. Apart from patients 9,10 , and 12 with two pathogenic $\mathrm{CNVs}$, we identified two patients (pt 39 and pt 46) with two rare potentially pathogenic changes. A complex clinical presentation in those patients supports a second-hit model, in which the compound effect of multiple CNVs, including those of unknown pathogenic significance, contributes to the phenotypic heterogeneity (Girirajan et al. 2012).

These results further demonstrate that array $\mathrm{CGH}$, in addition to the identification of CNVs and chromosomal aneuploidies, also enables the detection and estimation of their low-level mosaicism that may remain undetected by conventional cytogenetic methods (Cheung et al. 2007). Furthermore, our results support the usefulness of array CGH in the identification of the aneuploidy of cells under-represented in the T-cell population missed by routine chromosome analysis. However, apart from balanced aberrations (e.g., translocations, inversions), array CGH without SNP oligonucleotides cannot detect uniparental disomy.

In summary, we found that 69 of 256 patients with DD/ID carry one or more CNVs, in 38 cases responsible for the observed clinical features and in 13 patients potentially pathogenic for DD/ID. Our results further confirm the usefulness of array CGH in the detection of pathogenic CNVs in patients with idiopathic neurodevelopmental disorders.

Acknowledgments We are grateful to the patients and their families for participating in this study. We thank Drs. B.R. Brinkley, A.L. Beaudet, and J.R. Lupski for facilitating the collaboration between the Institute of Mother and Child and Baylor College of Medicine. The work was supported by grant R13-0005-04/2008 from the Polish Ministry of Science and Higher Education. M.B. is supported by the START fellowship from the Foundation for Polish Science.

Open Access This article is distributed under the terms of the Creative Commons Attribution License which permits any use, distribution, and reproduction in any medium, provided the original author(s) and the source are credited.

\section{References}

Ballif BC, Hornor SA, Jenkins E, Madan-Khetarpal S, Surti U, Jackson KE, Asamoah A, Brock PL, Gowans GC, Conway RL, Graham JM Jr, Medne L, Zackai EH, Shaikh TH, Geoghegan J, Selzer RR, Eis PS, Bejjani BA, Shaffer LG (2007) Discovery of a previously unrecognized microdeletion syndrome of 16p11.2-p12.2. Nat Genet 39:1071-1073

Battaglia A, Doccini V, Bernardini L, Novelli A, Loddo S, Capalbo A, Filippi T, Carey JC (2013) Confirmation of chromosomal 
microarray as a first-tier clinical diagnostic test for individuals with developmental delay, intellectual disability, autism spectrum disorders and dysmorphic features. Eur J Paediatr Neurol (in press)

Bonnet C, Andrieux J, Béri-Dexheimer M, Leheup B, Boute O, Manouvrier S, Delobel B, Copin H, Receveur A, Mathieu M, Thiriez G, Le Caignec C, David A, de Blois MC, Malan V, Philippe A, Cormier-Daire V, Colleaux L, Flori E, Dollfus H, Pelletier V, Thauvin-Robinet C, Masurel-Paulet A, Faivre L, Tardieu M, Bahi-Buisson N, Callier P, Mugneret F, Edery P, Jonveaux P, Sanlaville D (2010) Microdeletion at chromosome $4 q 21$ defines a new emerging syndrome with marked growth restriction, mental retardation and absent or severely delayed speech. J Med Genet 47:377-384

Boone PM, Bacino CA, Shaw CA, Eng PA, Hixson PM, Pursley AN, Kang SH, Yang Y, Wiszniewska J, Nowakowska BA, del Gaudio D, Xia Z, Simpson-Patel G, Immken LL, Gibson JB, Tsai AC, Bowers JA, Reimschisel TE, Schaaf CP, Potocki L, Scaglia F, Gambin T, Sykulski M, Bartnik M, Derwinska K, Wisniowiecka-Kowalnik B, Lalani SR, Probst FJ, Bi W, Beaudet AL, Patel A, Lupski JR, Cheung SW, Stankiewicz P (2010) Detection of clinically relevant exonic copy-number changes by array CGH. Hum Mutat 31:1326-1342

Bradley WE, Raelson JV, Dubois DY, Godin E, Fournier H, Privé C, Allard R, Pinchuk V, Lapalme M, Paulussen RJ, Belouchi A (2010) Hotspots of large rare deletions in the human genome. PLoS One 5: e9401

Burnside RD, Pasion R, Mikhail FM, Carroll AJ, Robin NH, Youngs EL, Gadi IK, Keitges E, Jaswaney VL, Papenhausen PR, Potluri VR, Risheg H, Rush B, Smith JL, Schwartz S, Tepperberg JH, Butler MG (2011) Microdeletion/microduplication of proximal $15 \mathrm{q} 11.2$ between BP1 and BP2: a susceptibility region for neurological dysfunction including developmental and language delay. Hum Genet 130:517-528

Cheung SW, Shaw CA, Scott DA, Patel A, Sahoo T, Bacino CA, Pursley A, Li J, Erickson R, Gropman AL, Miller DT, Seashore MR, Summers AM, Stankiewicz P, Chinault AC, Lupski JR, Beaudet AL, Sutton VR (2007) Microarray-based CGH detects chromosomal mosaicism not revealed by conventional cytogenetics. Am J Med Genet A 143A:1679-1686

Cooper GM, Coe BP, Girirajan S, Rosenfeld JA, Vu TH, Baker C, Williams C, Stalker H, Hamid R, Hannig V, Abdel-Hamid H, Bader P, McCracken E, Niyazov D, Leppig K, Thiese H, Hummel M, Alexander N, Gorski J, Kussmann J, Shashi V, Johnson K, Rehder C, Ballif BC, Shaffer LG, Eichler EE (2011) A copy number variation morbidity map of developmental delay. Nat Genet 43 : $838-846$

Cordoba I, González-Porras JR, Nomdedeu B, Luño E, de Paz R, Such E, Tormo M, Vallespi T, Collado R, Xicoy B, Andreu R, Muñoz JA, Solé F, Cervera J, del Cañizo C; Spanish Myelodysplastic Syndrome Registry (2012) Better prognosis for patients with del(7q) than for patients with monosomy 7 in myelodysplastic syndrome. Cancer 118:127-133

de Kovel CG, Trucks H, Helbig I, Mefford HC, Baker C, Leu C, Kluck C, Muhle H, von Spiczak S, Ostertag P, Obermeier T, Kleefuss-Lie AA, Hallmann K, Steffens M, Gaus V, Klein KM, Hamer HM, Rosenow F, Brilstra EH, Trenité DK, Swinkels ME, Weber YG, Unterberger I, Zimprich F, Urak L, Feucht M, Fuchs K, Møller RS, Hjalgrim H, De Jonghe P, Suls A, Rückert IM, Wichmann HE, Franke A, Schreiber S, Nürnberg P, Elger CE, Lerche H, Stephani U, Koeleman BP, Lindhout D, Eichler EE, Sander T (2010) Recurrent microdeletions at $15 \mathrm{q} 11.2$ and $16 \mathrm{p} 13.11$ predispose to idiopathic generalized epilepsies. Brain 133:23-32

Der Kaloustian VM, Russell L, Aradhya S, Richard G, Rosenblatt B, Melançon S (2011) A de novo 2.1-Mb deletion of 13q12.11 in a child with developmental delay and minor dysmorphic features. Am J Med Genet A 155A:2538-2542

Derwińska K, Bernaciak J, Wiśniowiecka-Kowalnik B, Obersztyn E, Bocian E, Stankiewicz P (2009) Autistic features with speech delay in a girl with an approximately $1.5-\mathrm{Mb}$ deletion in 6q16.1, including GPR63 and FUT9. Clin Genet 75:199-202

Derwińska K, Mierzewska H, Goszczańska A, Szczepanik E, Xia Z, Kuśmierska K, Tryfon J, Kutkowska-Kaźmierczak A, Bocian E, Mazurczak T, Obersztyn E, Stankiewicz P (2012) Clinical improvement of the aggressive neurobehavioral phenotype in a patient with a deletion of PITX3 and the absence of L-DOPA in the cerebrospinal fluid. Am J Med Genet B Neuropsychiatr Genet 159B:236-242

Dun XP, Chilton JK (2010) Control of cell shape and plasticity during development and disease by the actin-binding protein Drebrin. Histol Histopathol 25:533-540

Friedman JM, Baross A, Delaney AD, Ally A, Arbour L, Armstrong L, Asano J, Bailey DK, Barber S, Birch P, Brown-John M, Cao M, Chan S, Charest DL, Farnoud N, Fernandes N, Flibotte S, Go A, Gibson WT, Holt RA, Jones SJ, Kennedy GC, Krzywinski M, Langlois S, Li HI, McGillivray BC, Nayar T, Pugh TJ, RajcanSeparovic E, Schein JE, Schnerch A, Siddiqui A, Van Allen MI, Wilson G, Yong SL, Zahir F, Eydoux P, Marra MA (2006) Oligonucleotide microarray analysis of genomic imbalance in children with mental retardation. Am J Hum Genet 79:500-513

Girirajan S, Rosenfeld JA, Coe BP, Parikh S, Friedman N, Goldstein A, Filipink RA, McConnell JS, Angle B, Meschino WS, Nezarati MM, Asamoah A, Jackson KE, Gowans GC, Martin JA, Carmany EP, Stockton DW, Schnur RE, Penney LS, Martin DM, Raskin S, Leppig K, Thiese H, Smith R, Aberg E, Niyazov DM, Escobar LF, El-Khechen D, Johnson KD, Lebel RR, Siefkas K, Ball S, Shur N, McGuire M, Brasington CK, Spence JE, Martin LS, Clericuzio C, Ballif BC, Shaffer LG, Eichler EE (2012) Phenotypic heterogeneity of genomic disorders and rare copynumber variants. N Engl J Med 367:1321-1331

Grayton HM, Fernandes C, Rujescu D, Collier DA (2012) Copy number variations in neurodevelopmental disorders. Prog Neurobiol 99:81-91

Harada N, Nagai T, Shimokawa O, Niikawa N, Matsumoto N (2002) A $4 \mathrm{q} 21-\mathrm{q} 22$ deletion in a girl with severe growth retardation. Clin Genet 61:226-228

Harris JC (2006) Intellectual disability: understanding its development, causes, classification, evaluation, and treatment. Oxford University Press, Oxford

Huang N, Lee I, Marcotte EM, Hurles ME (2010) Characterising and predicting haploinsufficiency in the human genome. PLoS Genet 6: e1001154

Itsara A, Cooper GM, Baker C, Girirajan S, Li J, Absher D, Krauss RM, Myers RM, Ridker PM, Chasman DI, Mefford H, Ying P, Nickerson DA, Eichler EE (2009) Population analysis of large copy number variants and hotspots of human genetic disease. Am J Hum Genet $84: 148-161$

Kaminsky EB, Kaul V, Paschall J, Church DM, Bunke B, Kunig D, Moreno-De-Luca D, Moreno-De-Luca A, Mulle JG, Warren ST, Richard G, Compton JG, Fuller AE, Gliem TJ, Huang S, Collinson MN, Beal SJ, Ackley T, Pickering DL, Golden DM, Aston E, Whitby H, Shetty S, Rossi MR, Rudd MK, South ST, Brothman AR, Sanger WG, Iyer RK, Crolla JA, Thorland EC, Aradhya S, Ledbetter DH, Martin CL (2011) An evidence-based approach to establish the functional and clinical significance of copy number variants in intellectual and developmental disabilities. Genet Med 13:777-784

Koolen DA, Nillesen WM, Versteeg MH, Merkx GF, Knoers NV, Kets M, Vermeer S, van Ravenswaaij CM, de Kovel CG, Brunner HG, Smeets D, de Vries BB, Sistermans EA (2004) Screening for subtelomeric rearrangements in 210 patients with unexplained mental retardation using multiplex ligation dependent probe amplification (MLPA). J Med Genet 41:892-899

Koolen DA, Vissers LE, Pfundt R, de Leeuw N, Knight SJ, Regan R, Kooy RF, Reyniers E, Romano C, Fichera M, Schinzel A, Baumer A, Anderlid BM, Schoumans J, Knoers NV, van Kessel AG, Sistermans EA, Veltman JA, Brunner HG, de Vries BB (2006) A 
new chromosome 17q21.31 microdeletion syndrome associated with a common inversion polymorphism. Nat Genet 38:999-1001

Koolen DA, Pfundt R, de Leeuw N, Hehir-Kwa JY, Nillesen WM, Neefs I, Scheltinga I, Sistermans E, Smeets D, Brunner HG, van Kessel AG, Veltman JA, de Vries BB (2009) Genomic microarrays in mental retardation: a practical workflow for diagnostic applications. Hum Mutat 30:283-292

Lipska BS, Brzeskwiniewicz M, Wierzba J, Morzuchi L, Piotrowski A, Limon J (2011) 8.6Mb interstitial deletion of chromosome $4 q 13.3 q 21.23$ in a boy with cognitive impairment, short stature, hearing loss, skeletal abnormalities and facial dysmorphism. Genet Couns 22:353-363

Maulik PK, Mascarenhas MN, Mathers CD, Dua T, Saxena S (2011) Prevalence of intellectual disability: a meta-analysis of populationbased studies. Res Dev Disabil 32:419-436

Menten B, Maas N, Thienpont B, Buysse K, Vandesompele J, Melotte C, de Ravel T, Van Vooren S, Balikova I, Backx L, Janssens S, De Paepe A, De Moor B, Moreau Y, Marynen P, Fryns JP, Mortier G, Devriendt K, Speleman F, Vermeesch JR (2006) Emerging patterns of cryptic chromosomal imbalance in patients with idiopathic mental retardation and multiple congenital anomalies: a new series of 140 patients and review of published reports. J Med Genet 43:625-633

Miller DT, Adam MP, Aradhya S, Biesecker LG, Brothman AR, Carter NP, Church DM, Crolla JA, Eichler EE, Epstein CJ, Faucett WA, Feuk L, Friedman JM, Hamosh A, Jackson L, Kaminsky EB, Kok K, Krantz ID, Kuhn RM, Lee C, Ostell JM, Rosenberg C, Scherer SW, Spinner NB, Stavropoulos DJ, Tepperberg JH, Thorland EC, Vermeesch JR, Waggoner DJ, Watson MS, Martin CL, Ledbetter DH (2010) Consensus statement: chromosomal microarray is a firsttier clinical diagnostic test for individuals with developmental disabilities or congenital anomalies. Am J Hum Genet 86:749-764

Miyashita A, Arai H, Asada T, Imagawa M, Matsubara E, Shoji M, Higuchi S, Urakami K, Kakita A, Takahashi H, Toyabe S, Akazawa K, Kanazawa I, Ihara Y, Kuwano R; Japanese Genetic Study Consortium for Alzeheimer's Disease (2007) Genetic association of CTNNA3 with late-onset Alzheimer's disease in females. Hum Mol Genet 16:2854-2869

Morrow EM (2010) Genomic copy number variation in disorders of cognitive development. J Am Acad Child Adolesc Psychiatry 49: 1091-1104

Nowakowska BA, Obersztyn E, Szymańska K, Bekiesińska-Figatowska M, Xia Z, Ricks CB, Bocian E, Stockton DW, Szczałuba K, Nawara M, Patel A, Scott DA, Cheung SW, Bohan TP, Stankiewicz P (2010) Severe mental retardation, seizures, and hypotonia due to deletions of $M E F 2 C$. Am J Med Genet B Neuropsychiatr Genet 153B:10421051

Okajima T, Fukumoto S, Furukawa K, Urano T (1999) Molecular basis for the progeroid variant of Ehlers-Danlos syndrome. Identification and characterization of two mutations in galactosyltransferase I gene. J Biol Chem 274:28841-28844

Passon DM, Lee M, Fox AH, Bond CS (2011) Crystallization of a paraspeckle protein PSPC1-NONO heterodimer. Acta Crystallogr Sect F Struct Biol Cryst Commun 67:1231-1234

Pastorcic M, Das HK (2007) Analysis of transcriptional modulation of the presenilin 1 gene promoter by $Z N F 237$, a candidate binding partner of the Ets transcription factor ERM. Brain Res 1128:21-32

Patil SJ, Ponnala R, Shah S, Dalal A (2012) Mosaic trisomy 9 presenting with congenital heart disease, facial dysmorphism and pigmentary skin lesions: intricate issues of genetic counseling. Indian J Pediatr 79:806-809

Petti M, Samanich J, Pan Q, Huang CK, Reinmund J, Farooqi S, Morrow B, Babcock M (2011) Molecular characterization of an interstitial deletion of $1 \mathrm{p} 31.3$ in a patient with obesity and psychiatric illness and a review of the literature. Am J Med Genet A 155A:825-832

Pfundt R, Veltman JA (2012) Structural genomic variation in intellectual disability. Methods Mol Biol 838:77-95
Rauch A, Beese M, Mayatepek E, Dörr HG, Wenzel D, Reis A, Trautmann U (2003) A novel 5q35.3 subtelomeric deletion syndrome. Am J Med Genet A 121A:1-8

Rauch A, Hoyer J, Guth S, Zweier C, Kraus C, Becker C, Zenker M, Hüffmeier U, Thiel C, Rüschendorf F, Nürnberg P, Reis A, Trautmann U (2006) Diagnostic yield of various genetic approaches in patients with unexplained developmental delay or mental retardation. Am J Med Genet A 140:2063-2074

Ravnan JB, Tepperberg JH, Papenhausen P, Lamb AN, Hedrick J, Eash D, Ledbetter DH, Martin CL (2006) Subtelomere FISH analysis of 11688 cases: an evaluation of the frequency and pattern of subtelomere rearrangements in individuals with developmental disabilities. J Med Genet 43:478-489

Redon R, Baujat G, Sanlaville D, Le Merrer M, Vekemans M, Munnich A, Carter NP, Cormier-Daire V, Colleaux L (2006) Interstitial 9q22.3 microdeletion: clinical and molecular characterisation of a newly recognised overgrowth syndrome. Eur J Hum Genet 14:759767

Regier DA, Friedman JM, Marra CA (2010) Value for money? Array genomic hybridization for diagnostic testing for genetic causes of intellectual disability. Am J Hum Genet 86:765-772

Rodriguez-Revenga L, Mila M, Rosenberg C, Lamb A, Lee C (2007) Structural variation in the human genome: the impact of copy number variants on clinical diagnosis. Genet Med 9:600-606

Scollon S, McWalter K, Abe K, King J, Kimata K, Slavin TP (2012) Haploinsufficiency of STK11 and neighboring genes cause a contiguous gene syndrome including Peutz-Jeghers phenotype. Am J Med Genet A 158A:2959-2962

Shaffer LG, Theisen A, Bejjani BA, Ballif BC, Aylsworth AS, Lim C, McDonald M, Ellison JW, Kostiner D, Saitta S, Shaikh T (2007) The discovery of microdeletion syndromes in the post-genomic era: review of the methodology and characterization of a new 1q41q42 microdeletion syndrome. Genet Med 9:607-616

Sharp AJ, Hansen S, Selzer RR, Cheng Z, Regan R, Hurst JA, Stewart H, Price SM, Blair E, Hennekam RC, Fitzpatrick CA, Segraves R, Richmond TA, Guiver C, Albertson DG, Pinkel D, Eis PS, Schwartz S, Knight SJ, Eichler EE (2006) Discovery of previously unidentified genomic disorders from the duplication architecture of the human genome. Nat Genet 38:1038-1042

Sharp AJ, Selzer RR, Veltman JA, Gimelli S, Gimelli G, Striano P, Coppola A, Regan R, Price SM, Knoers NV, Eis PS, Brunner HG, Hennekam RC, Knight SJ, de Vries BB, Zuffardi O, Eichler EE (2007) Characterization of a recurrent $15 \mathrm{q} 24$ microdeletion syndrome. Hum Mol Genet 16:567-572

Sharp AJ, Mefford HC, Li K, Baker C, Skinner C, Stevenson RE, Schroer RJ, Novara F, De Gregori M, Ciccone R, Broomer A, Casuga I, Wang Y, Xiao C, Barbacioru C, Gimelli G, Bernardina BD, Torniero C, Giorda R, Regan R, Murday V, Mansour S, Fichera M, Castiglia L, Failla P, Ventura M, Jiang Z, Cooper GM, Knight SJ, Romano C, Zuffardi O, Chen C, Schwartz CE, Eichler EE (2008) A recurrent $15 \mathrm{q} 13.3$ microdeletion syndrome associated with mental retardation and seizures. Nat Genet 40:322-328

Shaw-Smith C, Pittman AM, Willatt L, Martin H, Rickman L, Gribble S, Curley R, Cumming S, Dunn C, Kalaitzopoulos D, Porter K, Prigmore E, Krepischi-Santos AC, Varela MC, Koiffmann CP, Lees AJ, Rosenberg C, Firth HV, de Silva R, Carter NP (2006) Microdeletion encompassing MAPT at chromosome 17q21.3 is associated with developmental delay and learning disability. Nat Genet 38:1032-1037

Shevell M, Ashwal S, Donley D, Flint J, Gingold M, Hirtz D, Majnemer A, Noetzel M, Sheth RD; Quality Standards Subcommittee of the American Academy of Neurology; Practice Committee of the Child Neurology Society (2003) Practice parameter: evaluation of the child with global developmental delay: report of the Quality Standards Subcommittee of the American Academy of Neurology 
and The Practice Committee of the Child Neurology Society. Neurology 60:367-380

Shim KS, Lubec G (2002) Drebrin, a dendritic spine protein, is manifold decreased in brains of patients with Alzheimer's disease and Down syndrome. Neurosci Lett 324:209-212

Slavotinek AM (2008) Novel microdeletion syndromes detected by chromosome microarrays. Hum Genet 124:1-17

Stankiewicz P, Beaudet AL (2007) Use of array CGH in the evaluation of dysmorphology, malformations, developmental delay, and idiopathic mental retardation. Curr Opin Genet Dev 17:182-192

Tanteles GA, Dixit A, Smith N, Martin K, Suri M (2011) Mild phenotype in a patient with a de-novo $2.9-\mathrm{Mb}$ interstitial deletion at $13 \mathrm{q} 12.11$. Clin Dysmorphol 20:61-65

Vergult S, Dauber A, Delle Chiaie B, Van Oudenhove E, Simon M, Rihani A, Loeys B, Hirschhorn J, Pfotenhauer J, Phillips JA 3rd, Mohammed S, Ogilvie C, Crolla J, Mortier G, Menten B (2012) 17q24.2 microdeletions: a new syndromal entity with intellectual disability, truncal obesity, mood swings and hallucinations. Eur J Hum Genet 20:534-539

Vissers LE, Stankiewicz P (2012) Microdeletion and microduplication syndromes. Methods Mol Biol 838:29-75

Vissers LE, de Vries BB, Veltman JA (2010) Genomic microarrays in mental retardation: from copy number variation to gene, from research to diagnosis. J Med Genet 47:289-297

Weiss LA, Arking DE; Gene Discovery Project of Johns Hopkins \& the Autism Consortium, Daly MJ, Chakravarti A (2009) A genomewide linkage and association scan reveals novel loci for autism. Nature 461:802-808

Wiszniewska J, Bi W, Shaw C, Stankiewicz P, Kang SH, Pursley AN, Lalani S, Hixson P, Gambin T, Tsai $\mathrm{CH}$, Bock $\mathrm{HG}$, Descartes M, Probst FJ, Scaglia F, Beaudet AL, Lupski JR, Eng C, Wai Cheung S, Bacino C, Patel A (2013) Combined array CGH plus SNP genome analyses in a single assay for optimized clinical testing. Eur J Hum Genet. doi:10.1038/ ejhg.2013.77 